\title{
Two-center holographic recording
}

\author{
Ali Adibi, ${ }^{*}$ Karsten Buse, ${ }^{\dagger}$ and Demetri Psaltis \\ Department of Electrical Engineering, California Institute of Technology, Pasadena, California 91125
}

Received March 6, 2000; revised manuscript received December 5, 2000

\begin{abstract}
We describe a two-center holographic recording method for the storage of persistent holograms in doubly doped lithium niobate crystals. We use a two-center model, and we show that our experimental observations can be explained by the model. We describe experimental methods for finding the unknown material parameters of $\mathrm{LiNbO}_{3}: \mathrm{Fe}: \mathrm{Mn}$ crystals for the two-center model, and we discuss the optimization of two-center recording. (C) 2001 Optical Society of America

OCIS codes: 090.0090, 090.2900, 090.7330.
\end{abstract}

\section{INTRODUCTION}

The type of recording material used remains the critical issue for holographic storage. Volume holographic storage systems with photorefractive crystals as the recording medium have been tried and tested. ${ }^{1-3}$ In such systems, the inhomogeneous illumination induced by the interference pattern of the reference and signal beams excites charge carriers from impurity levels into the conduction or valence bands; the charge carriers migrate, and they are trapped by empty impurities elsewhere. A spacecharge field builds up and modulates the refractive index by means of the electro-optic effect.

The photorefractive effect is reversible; i.e., homogeneous illumination redistributes the electrons, and new recording is possible. Thus read-write memories can be implemented. However, readout also requires homogeneous illumination at the recording wavelength, which erases the stored information. Thermal fixing, ${ }^{4}$ electrical fixing, ${ }^{5}$ two-photon recording, ${ }^{6}$ frequency-difference holograms, ${ }^{7}$ and readout with wave-vector spectra ${ }^{8}$ are known techniques for overcoming the problem of destructive readout. Of all these techniques, two-step (or twophoton) processes appear to be the most promising. They do not require heating or external electric fields, and they may permit recording with a high dynamic range, i.e., multiplexing of many holograms with high efficiency.

Two-step recording can be achieved in materials in which two photons are required for generation of one free electron or hole. The first photon excites the charge carrier into an intermediate level, and the second, into either the conduction or the valence band. Typically, different wavelengths are chosen for the first and the second excitations. Low-energy photons contain the holographic information, whereas high-energy photons sensitize the material for recording. During readout, only light of the recording wavelength is used, and persistent, nondestructive readout is achieved.

Multiphoton photorefractive storage has been discovered in lithium niobate $\left(\mathrm{LiNbO}_{3}\right)$ crystals, ${ }^{6}$ and persistent storage experiments utilizing two-photon excitations were performed with $\mathrm{LiTaO}_{3}$ which is isomorphic to $\mathrm{LiNbO}_{3} .{ }^{9}$ Picosecond light pulses (wavelengths of 1064 and $532 \mathrm{~nm}$ for the recording and sensitizing beams, respectively) were used in these early investigations. More recently, larger refractive-index changes and better sensitivities were achieved with $\mathrm{LiNbO}_{3}$ and nanosecond light pulses of the same wavelengths. ${ }^{10-12}$ The crystals were doped with iron or with copper as the photorefractive centers. The intermediate levels in all these experiments were due to $\mathrm{Nb}^{4+/ 5+}$ small polarons that are the result of intrinsic defect niobium on a lithium site $\left(\mathrm{Nb}_{\mathrm{Li}}\right){ }^{13,14}$ The recording sensitivity in the initial two-step recording experiments was extremely low owing to the short lifetime of the polarons in congruent $\mathrm{LiNbO}_{3}$. Therefore holograms we recorded by using high-intensity pulses. ${ }^{10-12}$

Two-step holographic recording with cw light was first performed in $\mathrm{LiNbO}_{3}: \operatorname{Pr}$ crystals, ${ }^{15-17}$ although it was later suggested that Pr does not play an important role in two-step recording in $\mathrm{LiNbO}_{3} .{ }^{18}$ More recently, it was shown that the lifetime of the shallow polaron levels can be considerably increased by use of highly reduced stoichiometric $\mathrm{LiNbO}_{3}$ crystals. ${ }^{18,19}$ Furthermore, reducing the $\mathrm{Fe}$ concentration (and eventually using nominally undoped stoichiometric $\mathrm{LiNbO}_{3}$ ) can further increase the lifetime of the polarons. Therefore, high recording and sensitizing light intensities are not required, and persistent holograms can be recorded in these crystals with $\mathrm{cw}$ light with much lower intensities ${ }^{18,19}$ than for previous pulsed experiments. The deeper traps in nominally undoped stoichiometric $\mathrm{LiNbO}_{3}$ are believed to be due to bipolarons. ${ }^{18,19}$ Despite impressive recent progress in the optimization of $\mathrm{LiNbO}_{3}$ crystals for this type of recording, sensitivity is still an important issue. Furthermore, using intrinsic levels for both shallower and deeper traps reduces control of the design and optimization of the material.

We recently showed ${ }^{20}$ that, by using doubly doped $\mathrm{LiNbO}_{3}$ crystals, we can obtain persistent holographic recording with better performance than from two-step recording. ${ }^{21}$ We refer to this technique as two-center holographic recording. In this paper we present a theoretical model and experimental verification of the model for two-center recording. The basic idea of two-center holographic recording is introduced in Section 2, and an experimental demonstration of the method is presented in Section 3. In Section 4 a theoretical model for the two- 
center method is introduced and compared with the experimental results. Based on the model developed in Section 4, the effects of the various design parameters on the holographic recording characteristics are investigated, an optimization scheme is developed in Section 5. The characteristics of the method along with some suggestions for improvement of its performance are discussed in Section 6. Final conclusions are made in Section 7.

\section{TWO-CENTER HOLOGRAPHIC RECORDING}

Two-center holographic recording is based on the use of doubly doped photochromic crystals, ${ }^{20}$ for example, $\mathrm{LiNbO}_{3}$ doped with manganese $(\mathrm{Mn})$ and iron $(\mathrm{Fe})$. It is known that $\mathrm{LiNbO}_{3}: \mathrm{Fe}: \mathrm{Mn}$ is photochromic and that UV preillumination enhances its sensitivity for a few recording and erasure cycles with visible light. ${ }^{22}$ The energyband diagram of such a crystal is shown in Fig. 1. Fe and $\mathrm{Mn}$ ions occur in the valence states $\mathrm{Mn}^{2+/ 3+}$ and $\mathrm{Fe}^{2+/ 3+}, 23$ and thermal depletion plays no role at room temperature. Electrons can be excited by UV light either from $\mathrm{Mn}^{2+}$ or from $\mathrm{Fe}^{2+}$ into the conduction band, whereas red light excites electrons only from the shallower $\mathrm{Fe}^{2+}$. The conduction-band electrons can recombine with both centers, and thus UV illumination populates the $\mathrm{Fe}^{2+/ 3+}$ level partially, whereas red-light illumination empties the Fe sites. The basic idea of twocenter holographic recording is to bring electrons from $\mathrm{Mn}$ to Fe with UV light via the conduction band, use these electrons to record the hologram with red light, and eventually transfer the spatial modulation of the electrons from Fe to Mn centers by red light. The result is a hologram stored in Mn centers that persists against further red illumination. One of the key material parameters in

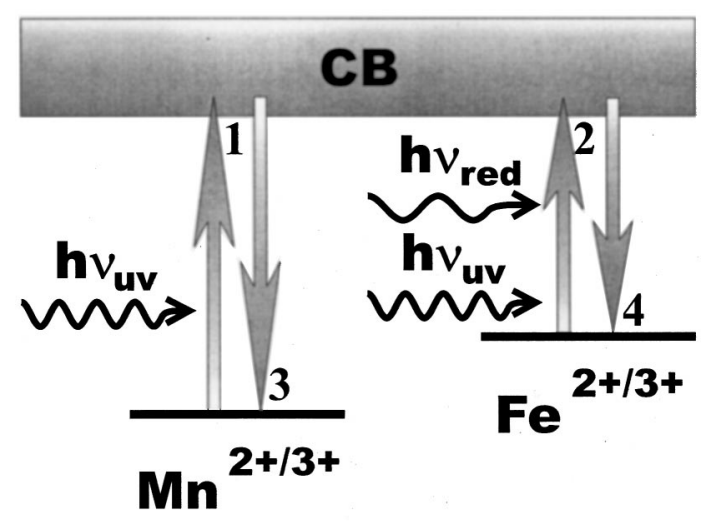

\section{VB}

Fig. 1. Energy-band diagram for a typical $\mathrm{LiNbO}_{3}$ crystal doped with Fe and Mn. CB and VB, conduction and valance bands, respectively. two-center holographic recording is the initial electron concentration in $\mathrm{Mn}$ and Fe traps. These concentrations can be varied by annealing (oxidation-reduction treatment). ${ }^{24}$ Inasmuch as Mn traps are deeper than Fe traps, electrons fill the Mn traps before the Fe traps. It is essential for persistent recording that all Fe traps initially be empty and that only a portion of the Mn traps be filled.

\section{EXPERIMENTS}

We performed experiments with a series of congruently melting $x$-cut $\mathrm{LiNbO}_{3}: \mathrm{Fe}: \mathrm{Mn}$ crystals with different doping levels and different annealing (or oxidationreduction) states. All characterization experiments were performed with a 0.85 -mm-thick $\mathrm{LiNbO}_{3}$ crystal doped with 0.075 wt. $\% \mathrm{Fe}_{2} \mathrm{O}_{3}$ and 0.01 wt. $\% \mathrm{MnO}$. The crystal was annealed appropriately such that all Fe traps as well as a portion of the Mn traps were empty. This annealing process results in negligible absorption at $633 \mathrm{~nm}$ and an intensity-absorption coefficient of $\alpha \simeq 9 \mathrm{~cm}^{-1}$ at $365 \mathrm{~nm}$ (the absorption spectrum of this crystal is similar to that of LN3 in Fig. 15 below). All other crystals were used in the optimization experiments.

\section{A. Experimental Setup}

The experimental setup is shown in Fig. 2(a). A homogeneous incoherent UV beam is used for sensitization, and two coherent red beams interfere at the crystal for holographic recording. The detailed experimental setup is shown in Fig. 2(b). We used a 100-W mercury lamp as the UV light source. The output light of the lamp is filtered (wavelength, 365 or $404 \mathrm{~nm}$, depending on the experiment) and focused by a lens to increase the UV intensity at the crystal. We used a $35-\mathrm{mW} \mathrm{He}-\mathrm{Ne}$ laser for generation of the coherent red light (wavelength, 633 $\mathrm{nm})$. By timing the opening and closing of shutters S1, S2, and S3 [Fig. 2(b)], we perform several experiments to help us to understand the physical mechanisms that are responsible for holographic recording and for optimizing the performance of the system.

\section{B. Sensitization and Bleaching Experiments}

A proper ratio between the intensities of the red recording and the UV sensitizing light, $I_{\text {red }} / I_{\mathrm{UV}}$, is essential for good performance. A convenient way to adjust the intensity ratio is to use sensitization and bleaching experiments.

To investigate sensitization dynamics, we monitor the absorption of the red light by the crystal. We do this by illuminating the crystal with a weak uniform red beam and monitoring the transmitted red power with time. The intensity of the illuminating red light should be low enough to have a negligible effect on the electron transfer between the traps. We perform the sensitization experiment with the experimental setup in Fig. 2(b) by opening shutters S1 and S2 and closing shutter S3. We monitor the bleaching dynamics by illuminating the sensitized crystal with only a strong uniform red beam and monitoring the transmitted power. We can do this by using the experimental setup in Fig. 2(b) by opening shutter S2 and closing shutters S1 and S3. Figure 3 shows typical re- 
(a)

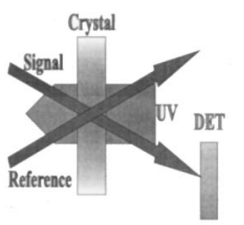

(b)

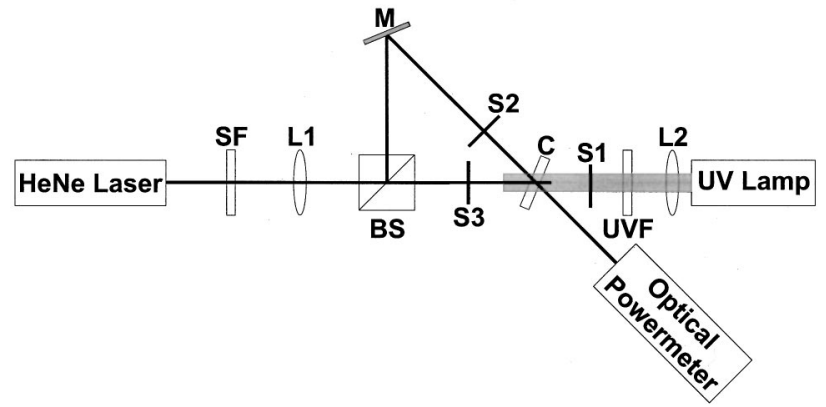

Fig. 2. Experimental setup for holographic recording experiments: (a) basic idea, (b) actual setup. SF, spatial filter; M, mirror; L1, L2, lenses; BS, beam splitter; S1-S3, shutters.
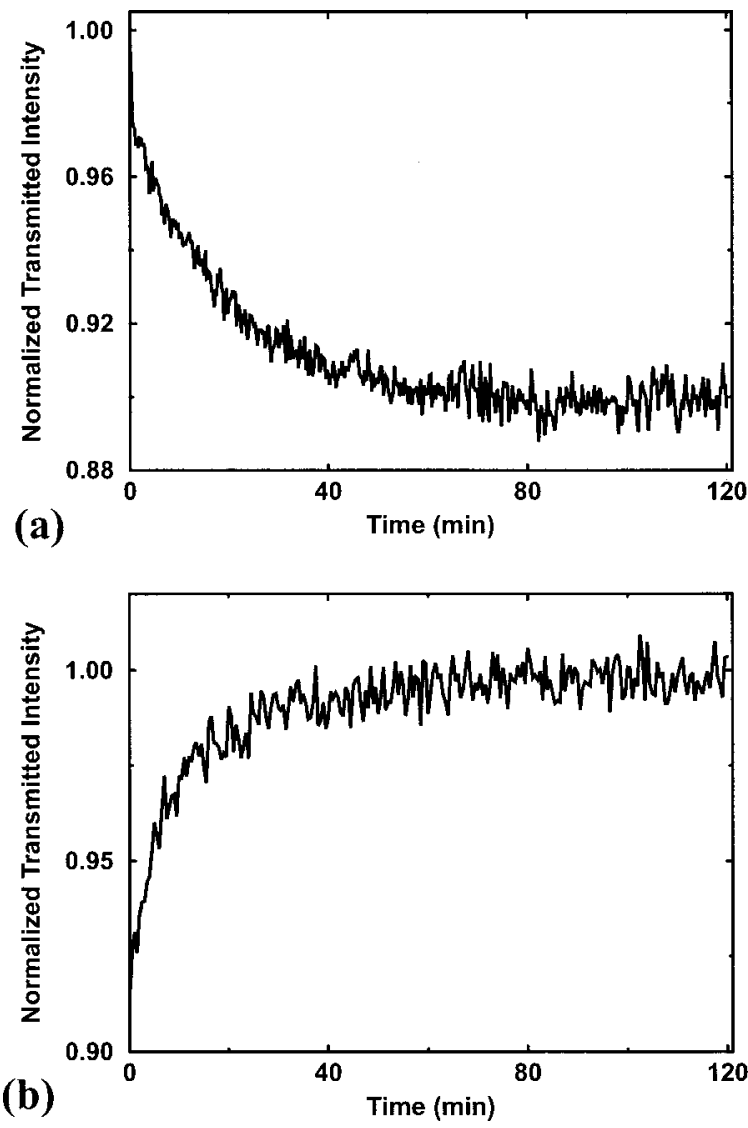

Fig. 3. Normalized transmitted red intensity in a $0.85-\mathrm{mm}$ $\mathrm{LiNbO}_{3}: \mathrm{Fe}: \mathrm{Mn}$ crystal. (a) Sensitization experiment: The crystal is sensitized with a homogeneous UV beam (wavelength, 365 $\mathrm{nm}$; intensity, $20 \mathrm{~mW} / \mathrm{cm}^{2}$ ) and monitored by a weak red beam (wavelength, $633 \mathrm{~nm}$; intensity, $0.6 \mathrm{~mW} / \mathrm{cm}^{2}$; ordinary polarization). (b) Bleaching experiment: The sensitized crystal is bleached with a strong red beam (wavelength, $633 \mathrm{~nm}$; intensity, $300 \mathrm{~mW} / \mathrm{cm}^{2}$; ordinary polarization). sults of the sensitization and bleaching experiments. The details of the experiments are summarized in the caption.

The time constants of sensitization and bleaching are measures of the rates of population and depopulation of the Fe sites. One can vary them by varying light intensities. These time constants should be of the same order of magnitude to produce a strongly modulated $\mathrm{Fe}^{2+}$ concentration during hologram recording. Excessive UV light (relative to the red light) results in too rapid sensitization and also too-rapid erasure of the hologram. Therefore, strong holograms cannot be recorded. However, red light that is too strong (compared with UV light) results in rapid bleaching of the $\mathrm{Fe}$ traps and in insufficient electrons in the Fe traps for efficient holographic recording. Therefore it is important to optimize the ratio between UV and red intensities. We find from the sensitization-bleaching experiments an optimum intensity ratio of $I_{\text {red }} / I_{\mathrm{UV}} \approx 25-30$ for recording the strongest hologram.

\section{Holographic Recording Experiments}

To get information about the holographic performance, we record and reconstruct plane-wave gratings, using the same $\mathrm{LiNbO}_{3}: \mathrm{Fe}: \mathrm{Mn}$ crystal that we used in the previous experiments. The unpolarized UV light illuminates the sample homogeneously; the $\mathrm{He}-\mathrm{Ne}$ laser light is split into two plane waves, which interfere at the crystal $\left(1 / e^{2}\right.$ beam diameter $2.0 \mathrm{~mm}$; transmission geometry; period length of the grating, $0.9 \mu \mathrm{m}$; intensity of each beam, 0.3 $\mathrm{W} / \mathrm{cm}^{2}$; ordinary polarization for both beams). The grating vector is aligned parallel to the $c$ axis of the sample. The crystal is preexposed to UV light for at least $3 \mathrm{~h}$ before recording. During recording, we block one of the $\mathrm{He}-\mathrm{Ne}$ beams [by closing $\mathrm{S} 2$ in Fig. 2(b)] from time to time, and the second beam is diffracted from the written grating to yield diffraction efficiency $\eta$ as the ratio between diffracted and total incident light intensities. Figure 4 shows the results. The diffraction efficiency rises quickly and then drops almost to zero with no UV light present [S1 closed in Fig. 2(b)] during the hologram formation. After some reading, the grating disappears completely. With the assistance of UV light during recording [S1 open in Fig. 2(b)], much higher efficiencies are obtained. Subsequent reading first erases the grating partially, but the remaining grating persists despite further red illumination. It is clear that the presence of UV light during hologram formation is crucial for obtaining large diffraction efficiencies and persistent readout. Finally, the hologram can be erased either by UV light only [S1 open, S2 and S3 closed in Fig. 2(b)] or by UV light and one of the red beams simultaneously [S1 and S3 open, S2 closed in Fig. 2(b)]. Figure 5 shows the diffraction efficiency versus time for a plane-wave hologram erased by UV and red beams simultaneously. The specifications of the beams are the same as those of the recording experiment.

\section{THEORY}

In this section we discuss a theoretical model that can explain the experimental results. The model is similar to 


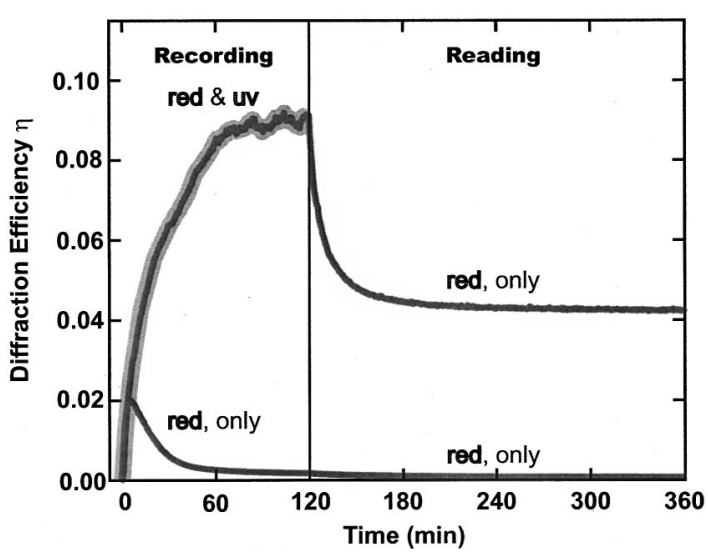

Fig. 4. Diffraction efficiency $\eta$ versus time for recording, without and with the simultaneous presence of UV light, and for subsequent reading in a $\mathrm{LiNbO}_{3}: \mathrm{Fe}: \mathrm{Mn}$ crystal.

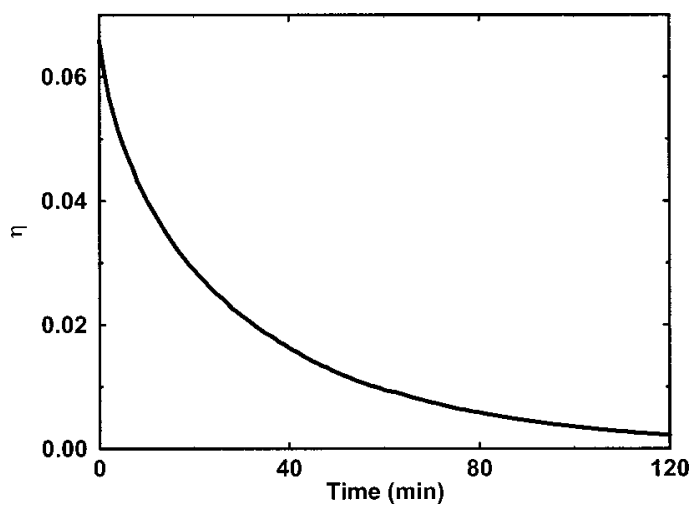

Fig. 5. Diffraction efficiency $\eta$ versus time for erasure with the simultaneous presence of UV and one of the red recording beams. The hologram was recorded by simultaneous presence of UV and two red recording beams to an arbitrary diffraction efficiency of nearly $7 \%$.

the two-center charge-transport model for $\mathrm{LiNbO}_{3}: \mathrm{Fe}$ introduced in 1993 by Jermann and Otten. ${ }^{25}$ The goal of the model is to find the time evolution of the space-charge field recorded by two interfering recording beams in the presence of a homogeneous sensitizing beam.

\section{A. Two-Center Model}

In the theoretical modeling of holographic recording in a doubly doped $\mathrm{LiNbO}_{3}$ :Fe:Mn crystal, we employ a set of five equations to solve for five unknowns. These unknowns are electron concentration in the conduction band $(n)$, electron concentration in the deeper and shallower traps $\left(N_{\mathrm{Mn}}{ }^{-}\right.$and $N_{\mathrm{Fe}}{ }^{-}$, respectively), current density $(j)$, and space-charge (electric) field $(E)$. The system of five equations is

$$
\begin{aligned}
\frac{\partial N_{\mathrm{Mn}}{ }^{-}}{\partial t}=- & -\left[q_{\mathrm{Mn}, R} s_{\mathrm{Mn}, R} I_{R}+q_{\mathrm{Mn}, \mathrm{UV}} s_{\mathrm{Mn}, \mathrm{UV}} I_{\mathrm{UV}}\right] N_{\mathrm{Mn}}{ }^{-} \\
& +\gamma_{\mathrm{Mn}} n\left(N_{\mathrm{Mn}}-N_{\mathrm{Mn}}{ }^{-}\right), \\
\frac{\partial N_{\mathrm{Fe}}{ }^{-}}{\partial t}=- & -\left[q_{\mathrm{Fe}, R} s_{\mathrm{Fe}, R} I_{R}+q_{\mathrm{Fe}, \mathrm{UV}} I_{\mathrm{UV}}\right] N_{\mathrm{Fe}}{ }^{-} \\
& +\gamma_{\mathrm{Fe}} n\left(N_{\mathrm{Fe}}-N_{\mathrm{Fe}}{ }^{-}\right),
\end{aligned}
$$

$$
\begin{aligned}
\frac{\partial j}{\partial x}= & e\left(\frac{\partial N_{\mathrm{Fe}}^{-}}{\partial t}+\frac{\partial N_{\mathrm{Mn}}^{-}}{\partial t}+\frac{\partial n}{\partial t}\right) \\
j= & e \mu n E+k_{B} T \mu \frac{\partial n}{\partial x} \\
& +\left(\kappa_{\mathrm{Fe}, R} I_{R}+\kappa_{\mathrm{Fe}, \mathrm{UV}} I_{\mathrm{UV}}\right) N_{\mathrm{Fe}}{ }^{-} \\
& +\left(\kappa_{\mathrm{Mn}, R} I_{R}+\kappa_{\mathrm{Mn}, \mathrm{UV}} I_{\mathrm{UV}}\right) N_{\mathrm{Mn}}{ }^{-}, \\
\frac{\partial E}{\partial x}= & \frac{\rho}{\epsilon \epsilon_{0}}=-\frac{e}{\epsilon \epsilon_{0}}\left(N_{\mathrm{Fe}}{ }^{-}+N_{\mathrm{Mn}}{ }^{-}+n-N_{A}\right) .
\end{aligned}
$$

All symbols are defined in Table 1 . Some parameters have a subscript $R$ or UV to indicate whether they are for red (in general, recording) or UV (in general, sensitizing) light. In writing Eqs. (1)-(5) we implicitly assumed that all variables have one-dimensional $(x)$ spatial variation.

Equations (1) and (2) are rate equations for the deeper $(\mathrm{Mn})$ and shallower (Fe) traps, respectively. Thermal depopulation of the traps is neglected, as both Fe and Mn traps are deep enough in the bandgap. Equation (3) is the current-continuity equation. Equation (4) is the current equation that describes the current density as the sum of drift, diffusion, and four bulk photovoltaic currents. Finally, Eq. (5) is Poisson's equation.

Equations (1)-(5) comprise a system of partial differential equations in time and space that is difficult to solve in general. To simplify the solution, we assume that the sensitizing intensity is homogeneous and that the recording intensity $\left(I_{R}\right)$ has one-dimensional sinusoidal variation with space:

$$
I_{R}=I_{R, 0}[1+m \cos (K x)],
$$

where $K$ and $m$ are the magnitude of the grating vector and the modulation depth of the recording intensity pattern, respectively. Therefore we represent each variable by the first two terms of its Fourier series. For example, the space-charge field $(E)$ can be represented as

$$
E=E_{0}+E_{1} \exp (i K x) .
$$

By replacing each variable in Eqs. (1)-(5) with the first two terms in its Fourier series, we can approximate Eqs. (1)-(5) by two sets of ordinary differential equations (for the zeroth- and first-order variables) that are much easier to solve numerically. To simplify the numerical solution, we assume that the steady state of the electron concentration in the conduction band $(n)$ is achieved instantaneously compared with the steady states of the other variables. This result is called adiabatic approximation and results in replacing $\partial n / \partial t=d n_{0} / d t=d n_{1} / d t=0$ in the governing equations. Performing simulations with and without this approximation results in essentially the same answer. Next, we assume that $n \ll N_{\mathrm{Mn}}{ }^{-}$, $N_{\mathrm{Fe}}{ }^{-}, N_{\mathrm{A}}$. This assumption results in omitting $n$ from Eq. (5). We also assume that there is no dc electric field; i.e., that $E_{0}=0$. This is true when we do not apply any external field to the crystal. The screening field is also negligible because of the presence of the UV beam and a considerable surface conductivity, as we discuss below. 
Table 1. Units, Meaning, and Values of All Quantities Involved in the Analysis of Two-Center Holographic Recording in a $\mathrm{LiNbO}_{3}: \mathrm{Fe}: \mathrm{Mn} \mathrm{Crystal}^{a}$

\begin{tabular}{|c|c|c|c|}
\hline Quantity (Unit) & Meaning & Value & Reference \\
\hline \multicolumn{4}{|l|}{ Parameter of $\mathrm{LiNbO}_{3}$} \\
\hline$r_{13}(\mathrm{~m} / \mathrm{V})$ & $\begin{array}{l}\text { Electro-optic coefficient (light wavelength, } 632.8 \\
\mathrm{~nm} \text { ) }\end{array}$ & $10.9 \times 10^{-12}$ & 28 \\
\hline$n_{0}$ & $\begin{array}{l}\text { Refractive index for ordinarily polarized light } \\
\text { (wavelength, } 632.8 \mathrm{~nm} \text { ) }\end{array}$ & 2.286 & 29 \\
\hline \multicolumn{4}{|c|}{ Charge-transport parameters } \\
\hline$j\left(\mathrm{~A} / \mathrm{m}^{2}\right)$ & Current density & Variable & \\
\hline$\mu\left(\mathrm{m}^{2} / \mathrm{Vs}\right)$ & Electron mobility in the conduction band & $7.4 \times 10^{-5}$ & 30 \\
\hline$n\left(\mathrm{~m}^{-3}\right)$ & Density of free electrons in the conduction band & Variable & \\
\hline$\rho\left(\mathrm{As} / \mathrm{m}^{3}\right)$ & Total charge density & Variable & \\
\hline$N_{A}\left(\mathrm{~m}^{-3}\right)$ & $\begin{array}{l}\text { Concentration of nonmobile positive compensation } \\
\text { charge, which maintains overall charge neutrality }\end{array}$ & $\left(3.4 \times 10^{24}\right)$ & \\
\hline$E(\mathrm{~V} / \mathrm{m})$ & Space-charge field & Variable & \\
\hline \multicolumn{4}{|l|}{ Fundamental constants } \\
\hline$k_{B}(\mathrm{~J} / \mathrm{K})$ & Boltzmann constant & $1.38 \times 10^{-23}$ & \\
\hline$\epsilon_{0}(\mathrm{As} / \mathrm{Vm})$ & Permittivity of free space & $8.85 \times 10^{-12}$ & \\
\hline \multicolumn{4}{|c|}{ Parameters related to the experimental conditions } \\
\hline$T(\mathrm{~K})$ & Crystal temperature & 293 & \\
\hline$K\left(\mathrm{~m}^{-1}\right)$ & Spatial frequency of the interference pattern & $6.9 \times 10^{6}$ & \\
\hline$\Lambda(\mathrm{m})$ & Period length of the interference pattern & $0.9 \times 10^{-6}$ & \\
\hline$I_{\mathrm{UV}}\left(\mathrm{W} / \mathrm{m}^{2}\right)$ & $\begin{array}{l}\text { Intensity of the spatially homogeneous UV light } \\
\text { (wavelength, } 365 \mathrm{~nm} \text { ) }\end{array}$ & Variable & \\
\hline$I_{R}\left(\mathrm{~W} / \mathrm{m}^{2}\right)$ & Intensity of the red light (wavelength, $633 \mathrm{~nm}$ ) & Variable & \\
\hline$m$ & $\begin{array}{l}\text { Modulation degree of the interference pattern of } \\
\text { the infrared light }\end{array}$ & Variable & \\
\hline \multicolumn{4}{|l|}{ Parameters of $\mathrm{Fe}$} \\
\hline$N_{\mathrm{Fe}}\left(\mathrm{m}^{-3}\right)$ & Total concentration of $\mathrm{Fe}$ & $\left(2.5 \times 10^{25}\right)$ & \\
\hline$N_{\mathrm{Fe}}^{-}\left(\mathrm{m}^{-3}\right)$ & Concentration of $\mathrm{Fe}^{2+}$ & $(0)$ & \\
\hline$s_{\mathrm{Fe}, R}\left(\mathrm{~m}^{2} / \mathrm{J}\right)$ & $\begin{array}{l}\text { Photon absorption cross section of } \mathrm{Fe}^{2+} \text { (light } \\
\text { wavelength, } 633 \mathrm{~nm} \text { ) }\end{array}$ & $3.7 \times 10^{-4}$ & 31,32 \\
\hline$q_{\mathrm{Fe}, R} s_{\mathrm{Fe}, R}\left(\mathrm{~m}^{2} / \mathrm{J}\right)$ & $\begin{array}{l}\text { Absorption cross section of } \mathrm{Fe}^{2+} \text { for absorption of } \\
\text { a photon and excitation of an electron from } \mathrm{Fe}^{2+} \\
\text { into the conduction band (light wavelength, } 633 \\
\mathrm{~nm} \text { ) }\end{array}$ & $3.3 \times 10^{-6}$ & 33 \\
\hline$q_{\mathrm{Fe}, \mathrm{UV}} s_{\mathrm{Fe}, \mathrm{UV}}\left(\mathrm{m}^{2} / \mathrm{J}\right)$ & $\begin{array}{l}\text { Absorption cross section of } \mathrm{Fe}^{2+} \text { for absorption of } \\
\text { a photon and excitation of an electron from } \mathrm{Fe}^{2+} \\
\text { into the conduction band (light wavelength, } 365 \\
\mathrm{~nm} \text { ) }\end{array}$ & $3.8 \times 10^{-5}$ & 33 \\
\hline$\gamma_{\mathrm{Fe}}\left(\mathrm{m}^{3} / \mathrm{s}\right)$ & $\begin{array}{l}\text { Coefficient for recombination of conduction-band } \\
\text { electrons with } \mathrm{Fe}^{3+}\end{array}$ & $1.65 \times 10^{-14}$ & 25 \\
\hline$-\kappa_{\mathrm{Fe}, R}\left(\mathrm{~m}^{3} / \mathrm{V}\right)$ & $\begin{array}{l}\text { Bulk photovoltaic coefficient for excitation of electrons } \\
\text { from } \mathrm{Fe}^{2+} \text { into the conduction band (light } \\
\text { wavelength, } 633 \mathrm{~nm} \text { ) }\end{array}$ & $7 \times 10^{-34}$ & 31,33 \\
\hline$-\kappa_{\mathrm{Fe}, \mathrm{UV}}\left(\mathrm{m}^{3} / \mathrm{V}\right)$ & $\begin{array}{l}\text { Bulk photovoltaic coefficient for excitation of electrons } \\
\text { from } \mathrm{Fe}^{2+} \text { into the conduction band (light } \\
\text { wavelength, } 365 \mathrm{~nm} \text { ) }\end{array}$ & $1.4 \times 10^{-32}$ & 32,33 \\
\hline \multicolumn{4}{|c|}{ 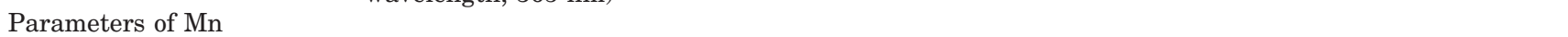 } \\
\hline$N_{\mathrm{Mn}}\left(\mathrm{m}^{-3}\right)$ & Total concentration of $\mathrm{Mn}$ & $\left(3.8 \times 10^{24}\right)$ & \\
\hline$N_{\mathrm{Mn}}^{-}\left(\mathrm{m}^{-3}\right)$ & Concentration of $\mathrm{Mn}^{2+}$ & $\left(3.4 \times 10^{24}\right)$ & \\
\hline$q_{\mathrm{Mn}, R} s_{\mathrm{Mn}, R}\left(\mathrm{~m}^{2} / \mathrm{J}\right)$ & $\begin{array}{l}\text { Absorption cross section of } \mathrm{Mn}^{2+} \text { for absorption } \\
\text { of a photon and excitation of an electron into the } \\
\text { conduction band (light wavelength, } 633 \mathrm{~nm} \text { ) }\end{array}$ & $(0)$ & \\
\hline$q_{\mathrm{Mn}, \mathrm{UV}} s_{\mathrm{Mn}, \mathrm{UV}}\left(\mathrm{m}^{2} / \mathrm{J}\right)$ & $\begin{array}{l}\text { Absorption cross section of } \mathrm{Mn}^{2+} \text { for absorption } \\
\text { of a photon and excitation of an electron into the } \\
\text { conduction band (light wavelength, } 365 \mathrm{~nm} \text { ) }\end{array}$ & $3.6 \times 10^{-5}$ & This work \\
\hline$\gamma_{\mathrm{Mn}}\left(\mathrm{m}^{3} / \mathrm{s}\right)$ & $\begin{array}{l}\text { Coefficient for recombination of conduction-band } \\
\text { electrons with } \mathrm{Mn}^{3+}\end{array}$ & $2.4 \times 10^{-13}$ & This work \\
\hline$-\kappa_{\mathrm{Mn}, R}\left(\mathrm{~m}^{3} / \mathrm{V}\right)$ & $\begin{array}{l}\text { Bulk photovoltaic coefficient for excitation of electrons } \\
\text { from } \mathrm{Mn}^{2+} \text { into the conduction band (light } \\
\text { wavelength, } 633 \mathrm{~nm} \text { ) }\end{array}$ & -2 & \\
\hline$-\kappa_{\mathrm{Mn}, \mathrm{UV}}\left(\mathrm{m}^{3} / \mathrm{V}\right)$ & $\begin{array}{l}\text { Bulk photovoltaic coefficient for excitation of electrons } \\
\text { from } \mathrm{Mn}^{2+} \text { into the conduction band (light } \\
\text { wavelength, } 365 \mathrm{~nm} \text { ) }\end{array}$ & $1.1 \times 10^{-32}$ & This work \\
\hline
\end{tabular}

${ }^{a}$ Subscripts 0 and 1 are added in the text to the spatially dependent quantities to indicate zeroth- and first-order Fourier components. Values in parentheses are standard values, which are valid if no other value is mentioned. Most of the values are determined by use of the experimental data curves in the referenced literature. 
After finding $E_{1}$ by solving the governing equations numerically, we can easily calculate the change in the index of refraction through the electro-optic effect as

$$
\Delta n=-\left(n_{\mathrm{eff}}^{3} / 2\right) r_{\mathrm{eff}} E_{1},
$$

where $r_{\text {eff }}$ is the effective electro-optic coefficient, which depends on the direction of the space-charge field with respect to the $c$ axis of the crystal and on the polarization of the readout beam, and $n_{\text {eff }}$ is the effective index of refraction for the readout beam, which depends on the polarization of that beam. Finally, we can calculate the diffraction efficiency from $\Delta n$ by using Kogelnik's formula ${ }^{34}$ :

$$
\eta=\sin ^{2}\left(\frac{\pi \Delta n L}{\lambda \cos \theta}\right),
$$

where $L, \lambda$, and $\theta$ are the material thickness, the wavelength of the recording (and reading) beams in vacuum, and the angle between the normal to the crystal and each recording beam (or half of the angle between the two recording beams) inside the crystal, respectively.

\section{B. Parameters of the Model}

To have a reliable model we need accurate estimates of the parameters. Although most of the parameters for $\mathrm{Fe}$ traps can be extracted from the literature, important parameters for the Mn traps and the initial electron concentration in the Mn traps $\left(N_{A}\right)$ need to be determined.

Knowing all Fe parameters, we performed experiments to find three additional parameters: $q_{\mathrm{Mn}, \mathrm{UV}} s_{\mathrm{Mn}, \mathrm{UV}}, \gamma_{\mathrm{Mn}}$, and the initial electron concentration in the Mn traps $\left[N_{\mathrm{Mn} 0}{ }^{-}(t=0)=N_{A}\right]$. We can assume that $q_{\mathrm{Mn}, R} s_{\mathrm{Mn}, R}$ and $\kappa_{\mathrm{Mn}, R}$ are zero because of the deep position of the Mn traps in the bandgap of $\mathrm{LiNbO}_{3}$. To find the three parameters mentioned above we need to obtain three equations. We use bleaching and sensitization experiments to find these equations. After finding these three parameters, we use curve fitting to the measured holographic recording curves to find the last unknown parameter, $\kappa_{\mathrm{Mn}, \mathrm{UV}}$. Finally, we optimize our calculation of the parameters by fine tuning these parameters to get the best fit to the sensitization, bleaching, and holographic recording curves. The important parameters are listed in Table 1.

\section{Bleaching}

We use the bleaching experiment to obtain a relationship between $\gamma_{\mathrm{Mn}}$ and $N_{A}$. The governing equations for the bleaching dynamics are

$$
\begin{aligned}
& \frac{\partial N_{\mathrm{Mn}}{ }^{-}}{\partial t}=\gamma_{\mathrm{Mn}} n\left(N_{\mathrm{Mn}}-N_{\mathrm{Mn}^{-}}\right), \\
& \frac{\partial N_{\mathrm{Fe}}^{-}}{\partial t}=-q_{\mathrm{Fe}, R} s_{\mathrm{Fe}, R} I_{R} N_{\mathrm{Fe}}{ }^{-} \\
&+\gamma_{\mathrm{Fe}} n\left(N_{\mathrm{Fe}}-N_{\mathrm{Fe}}{ }^{-}\right), \\
& \frac{\partial N_{\mathrm{Fe}}{ }^{-}}{\partial t}+\frac{\partial N_{\mathrm{Mn}}{ }^{-}}{\partial t}=\frac{1}{e} \frac{\partial j}{\partial x}=0,
\end{aligned}
$$

where we assume adiabatic approximation $(\partial n / \partial t=0)$ in Eq. (12). The initial conditions for $N_{\mathrm{Fe}}{ }^{-}$and $N_{\mathrm{Mn}}{ }^{-}$de- pend on the sensitization process, but the condition $N_{\mathrm{Fe}}{ }^{-}+N_{\mathrm{Mn}}{ }^{-}=N_{A}$ must always be satisfied. We can find $n$ as a function of $N_{\mathrm{Mn}}{ }^{-}$and $N_{\mathrm{Fe}}{ }^{-}$by substituting $\partial N_{\mathrm{Mn}}{ }^{-} / \partial t$ and $\partial N_{\mathrm{Fe}^{-}}{ }^{-} / \partial t$ from Eqs. (10) and (11), respectively, into Eq. (12). Substituting $N_{\mathrm{Mn}}{ }^{-}=N_{A}-N_{\mathrm{Fe}}{ }^{-}$ and the formula for $n$ into Eq. (11), we can obtain a differential equation for $N_{\mathrm{Fe}}{ }^{-}$as

$$
\begin{aligned}
\frac{\partial N_{\mathrm{Fe}}{ }^{-}(z)}{\partial t} & \\
& =-\frac{q_{\mathrm{Fe}, R} s_{\mathrm{Fe}, R} I_{R} \gamma_{\mathrm{Mn}}\left(N_{\mathrm{Mn}}-N_{\mathrm{Mn}}{ }^{-}\right)}{\gamma_{\mathrm{Mn}}\left(N_{\mathrm{Mn}}-N_{\mathrm{Mn}}{ }^{-}\right)+\gamma_{\mathrm{Fe}}\left(N_{\mathrm{Fe}}-N_{\mathrm{Fe}}{ }^{-}\right)} N_{\mathrm{Fe}}{ }^{-} \\
& =-\frac{N_{\mathrm{Fe}}{ }^{-}(z)}{\tau_{b}(t, z)} .
\end{aligned}
$$

To simplify the calculations, we assume that the absorption of the red light in the crystal is weak. Therefore we can consider the red light intensity $\left(I_{R}\right)$ in Eq. (13) to be a constant. We further assume that $N_{\mathrm{Fe}}{ }^{-} \ll N_{\mathrm{Fe}}$ and $N_{\mathrm{Mn}}{ }^{-} \simeq N_{A}$. This is a good approximation when we neglect the beginning of the bleaching curve [Fig. 3(b)] and consider the dynamics of the latter part of the curve. With all these approximations, bleaching time constant $\tau_{b}$ in Eq. (13) becomes a constant, independently of position inside the crystal. The bleaching speed normalized to the bleaching light intensity $\left(I_{R}\right)$ can then be written as

$$
\frac{1}{\tau_{b} I_{R}}=\left(q_{\mathrm{Fe}, R} s_{\mathrm{Fe}, R}\right)\left[1+\frac{\gamma_{\mathrm{Fe}} N_{\mathrm{Fe}}}{\gamma_{\mathrm{Mn}}\left(N_{\mathrm{Mn}}-N_{A}\right)}\right]^{-1} .
$$

We can find the left-hand side of Eq. (14) experimentally by fitting the latter parts of the bleaching curves at different intensities with monoexponential formulas. We need to find the relation between light transmission and electron concentration in Fe traps $\left(N_{\mathrm{Fe}}{ }^{-}\right)$. For small absorption of the bleaching light inside a crystal with thickness $L$, we can write

$$
I_{t}=I_{i} \exp \left[-\int_{0}^{L} \alpha(z) \mathrm{d} z\right] \simeq I_{i}\left[1-\int_{0}^{L} \alpha(z) \mathrm{d} z\right],
$$

where

$$
\alpha(z)=\left(s_{\mathrm{Fe}, R} h \nu\right) N_{\mathrm{Fe}}{ }^{-}(z),
$$

and $I_{t}$ and $I_{i}$ are the transmitted and incident intensities [or $I(z=L)$ and $I(z=0)$ ], respectively. Therefore the bleaching time constant is equal to time constant $\tau_{b}$ in the variation of $\mathrm{NFe}_{\mathrm{Fe}}{ }^{-}$and we can measure $\left(1 / \tau_{b}\right) / I_{R}$ from the bleaching experiments and use it in Eq. (14). Figure 6 shows the variation of the bleaching speed $\left(1 / \tau_{b}\right)$ with red-light intensity $\left(I_{R}\right)$. The solid line in Fig. 6 shows the linear fit to the experimental data. Using the slope of this line, we can obtain one equation for the unknown parameters:

$$
\frac{\gamma_{\mathrm{Fe}} N_{\mathrm{Fe}}}{\gamma_{\mathrm{Mn}}\left(N_{\mathrm{Mn}}-N_{A}\right)}=3.44
$$

\section{Sensitization}

We use the measurements from the sensitization experiment to obtain two additional relationships for the three 


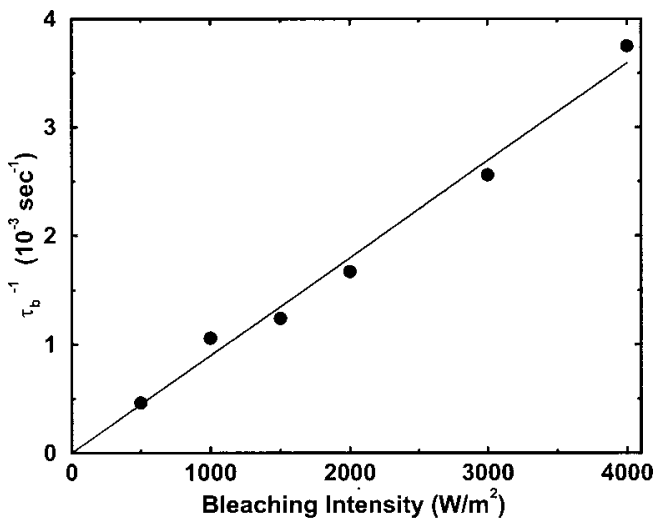

Fig. 6. Variation of bleaching speed $\left(1 / \tau_{b}\right)$ with bleaching intensity $\left(I_{R}\right)$. Solid line, linear fit to the experimental data.

unknowns $\left(q_{\mathrm{Mn}, \mathrm{UV}} s_{\mathrm{Mn}, \mathrm{UV}}, \gamma_{\mathrm{Mn}}\right.$, and $\left.N_{A}\right)$. The governing equations for the analysis of the sensitization dynamics are

$$
\begin{aligned}
\frac{\partial N_{\mathrm{Mn}}{ }^{-}}{\partial t}= & -q_{\mathrm{Mn}, \mathrm{UV}} s_{\mathrm{Mn}, \mathrm{UV}} I_{\mathrm{UV}} N_{\mathrm{Mn}}{ }^{-} \\
& +\gamma_{\mathrm{Mn}} n\left(N_{\mathrm{Mn}}-N_{\mathrm{Mn}}{ }^{-}\right), \\
\frac{\partial N_{\mathrm{Fe}}{ }^{-}}{\partial t}= & -q_{\mathrm{Fe}, \mathrm{UV}} s_{\mathrm{Fe}, \mathrm{UV}} I_{\mathrm{UV}} N_{\mathrm{Fe}}{ }^{-} \\
& +\gamma_{\mathrm{Fe}} n\left(N_{\mathrm{Fe}}-N_{\mathrm{Fe}}{ }^{-}\right), \\
\frac{\partial N_{\mathrm{Fe}}{ }^{-}}{\partial t}+\frac{\partial N_{\mathrm{Mn}}{ }^{-}}{\partial t}= & \frac{1}{e} \frac{\partial j}{\partial x}=0,
\end{aligned}
$$

where we assume the adiabatic approximation $(\partial n / \partial t$ $=0)$ in Eq. (20). The initial conditions are $\mathrm{N}_{\mathrm{Fe}}{ }^{-}(t$ $=0)=0$ and $N_{\mathrm{Mn}}{ }^{-}(t=0)=N_{A}$. Using $N_{\mathrm{Mn}}{ }^{-}=N_{A}$ - $N_{\mathrm{Fe}}{ }^{-}$from Eq. (20), we can find the differential equation for the electron concentration in the Fe traps as
Note that the initial conditions for the sensitization experiment are that $N_{\mathrm{Fe}}{ }^{-}(t=0)=0$ and $N_{\mathrm{Mn}}{ }^{-}(t=0)$ $=N_{A}$.

The left-hand side of Eq. (23) can be calculated from the experimental results. Figure 7 (a) shows the variation of the initial sensitization slope $\left[\left|\mathrm{d}\left(I_{t} / I_{i}\right) / \mathrm{d} t\right|_{t=0} \mid\right]$ with $I_{\mathrm{UV} 0}$. Figure 7(a) shows that the initial sensitization slope varies linearly with the UV intensity $I_{\mathrm{UV}}$, as Eq. (23) suggests. Replacing the slope of the line fitted to the experimental data $\left[\left|\mathrm{d}\left(I_{t} / I_{i}\right) / \mathrm{d} t\right|_{t=0} \mid / I_{\mathrm{UV} 0}\right]$ in Eq. (23) results in

$$
\frac{\left(s_{\mathrm{Fe}, R} h \nu\right) q_{\mathrm{Mn}, \mathrm{UV}} s_{\mathrm{Mn}, \mathrm{UV}} N_{A}}{1+\frac{\gamma_{\mathrm{Mn}}\left(N_{\mathrm{Mn}}-N_{A}\right)}{\gamma_{\mathrm{Fe}} N_{\mathrm{Fe}}}} \frac{1}{\alpha_{\mathrm{UV}}}=1.06 \times 10^{-6} .
$$

A second equation can be obtained from use of the saturation value of the transmitted red light in the sensitization experiment. Figure $7(\mathrm{~b})$ shows the ratio of the transmitted to the incident red intensity after $3 \mathrm{~h}$ of sensitization as a function of UV intensity $I_{\mathrm{UV}}$. This value is related to the electron concentration in Fe traps after 3 $\mathrm{h}$ of sensitization. The relation between this transmitted-to-incident intensity ratio and the final (steady-state) electron concentration in the $\mathrm{Fe}$ traps, $N_{\mathrm{Fe}, \text { final }}{ }^{-}$, is derived in $\mathbf{A}$. Therefore we can find $N_{\mathrm{Fe}, \text { final }}$ from Fig. 7(b) (see Appendix A). Putting the steady-state conditions $\partial N_{\mathrm{Fe}}{ }^{-} / \partial t=0$ and $N_{\mathrm{Fe}}{ }^{-}=N_{\mathrm{Fe} \text {,final }}{ }^{-}$into Eq. (21), we obtain the following relation between $N_{A}$ and $q_{\mathrm{Mn}, \mathrm{UV}} s_{\mathrm{Mn}, \mathrm{UV}}$ :

$$
\begin{aligned}
& q_{\mathrm{Fe}, \mathrm{UV}} s_{\mathrm{Fe}, \mathrm{UV}} \gamma_{\mathrm{Mn}}\left(N_{\mathrm{Mn}}-N_{A}+N_{\mathrm{Fe}, \mathrm{final}}{ }^{-}\right) N_{\mathrm{Fe}, \mathrm{final}}{ }^{-} \\
& \quad=\gamma_{\mathrm{Fe}} q_{\mathrm{Mn}, \mathrm{UV}} s_{\mathrm{Mn}, \mathrm{UV}}\left(N_{\mathrm{Fe}}-N_{\mathrm{Fe}, \text { final }}{ }^{-}\right)\left(N_{A}-N_{\mathrm{Fe}, \text { final }}{ }^{-}\right) .
\end{aligned}
$$

\section{Finding the Unknown Parameters}

$q_{M n, U V} S_{M n, U V}, \gamma_{M n}$, and $N_{A}$

The three unknowns $\left(q_{\mathrm{Mn}, \mathrm{UV}} s_{\mathrm{Mn}, \mathrm{UV}}, \gamma_{\mathrm{Mn}}\right.$, and $\left.N_{A}\right)$ can be

$\frac{\partial N_{\mathrm{Fe}}{ }^{-}}{\partial t}=\frac{-q_{\mathrm{Fe}, \mathrm{UV}} s_{\mathrm{Fe}, \mathrm{UV}} \gamma_{\mathrm{Mn}}\left(N_{\mathrm{Mn}}-N_{A}+N_{\mathrm{Fe}}{ }^{-}\right) N_{\mathrm{Fe}}{ }^{-}+\gamma_{\mathrm{Fe}} q_{\mathrm{Mn}, \mathrm{UV}} s_{\mathrm{Mn}, \mathrm{UV}}\left(N_{\mathrm{Fe}}-N_{\mathrm{Fe}}{ }^{-}\right)\left(N_{A}-N_{\mathrm{Fe}}{ }^{-}\right)}{\gamma_{\mathrm{Fe}}\left(N_{\mathrm{Fe}}-N_{\mathrm{Fe}}{ }^{-}\right)+\gamma_{\mathrm{Mn}}\left(N_{\mathrm{Mn}}-N_{A}+N_{\mathrm{Fe}}{ }^{-}\right)} I_{\mathrm{UV}}$.

The major complication in finding an analytic solution for Eq. (21) is the strong absorption of UV light. The measured absorption coefficient of the crystal at $365 \mathrm{~nm}$ is $\alpha=9 \mathrm{~mm}^{-1}$. Differentiating both sides of Eq. (15) with respect to time, we can calculate the initial slope of the transmitted intensity ratio versus time as

$$
\left.\frac{\mathrm{d}\left(I_{t} / I_{i}\right)}{\mathrm{d} t}\right|_{t=0}=-\left.s_{\mathrm{Fe}, R} h \nu \int_{0}^{L} \frac{\mathrm{d} N_{\mathrm{Fe}}^{-}(z)}{\mathrm{d} t}\right|_{t=0} \mathrm{~d} z
$$

Substituting $\left.\left[\mathrm{d} N_{\mathrm{Fe}^{-}}{ }^{-}(z) / \mathrm{d} t\right]\right|_{t=0}$ from Eq. (21) into Eq. (22) and replacing $I_{\mathrm{UV}}(z)$ by $I_{\mathrm{UV} 0} \exp \left(-\alpha_{\mathrm{UV}} z\right)$, we obtain

$$
\left|\frac{\mathrm{d}\left(I_{t} / I_{i}\right)}{\mathrm{d} t}\right|_{t=0} \mid=\frac{\left(s_{\mathrm{Fe}, R} h \nu\right) q_{\mathrm{Mn}, \mathrm{UV}} s_{\mathrm{Mn}, \mathrm{UV}} N_{A}}{1+\frac{\gamma_{\mathrm{Mn}}\left(N_{\mathrm{Mn}}-N_{A}\right)}{\gamma_{\mathrm{Fe}} N_{\mathrm{Fe}}}} \frac{I_{\mathrm{UV} 0}}{\alpha_{\mathrm{UV}}}
$$

found by solving the system of Eqs. (17), (24), and (25) This system of equations has two sets of solutions. However, one set of values for $q_{\mathrm{Mn}, \mathrm{UV}} s_{\mathrm{Mn}, \mathrm{UV}}, \gamma_{\mathrm{Mn}}$, and $N_{A}$ does not agree with the experimental results. The acceptable set of values is

$$
\begin{aligned}
N_{A} & =3.1 \times 10^{24} \mathrm{~m}^{-3}, \\
q_{\mathrm{Mn}, \mathrm{UV}} s_{\mathrm{Mn}, \mathrm{UV}} & =3.55 \times 10^{-5} \mathrm{~m}^{2} / \mathrm{J} \\
\gamma_{\mathrm{Mn}} & =8.5 \gamma_{\mathrm{Fe}}=1.32 \times 10^{-13} \mathrm{~m}^{-3} \mathrm{~s}^{-1} .
\end{aligned}
$$

We use $N_{A}, q_{\mathrm{Mn}, \mathrm{UV}} s_{\mathrm{Mn}, \mathrm{UV}}$, and $\gamma_{\mathrm{Mn}}$ from Eqs. (26)-(28) as the initial values in the simulation of sensitization, bleaching, and holographic recording and readout curves and then fine tune these values by trying to get the best fits to the experimental results. The only remaining unknown parameter is the photovoltaic constant of the Mn 

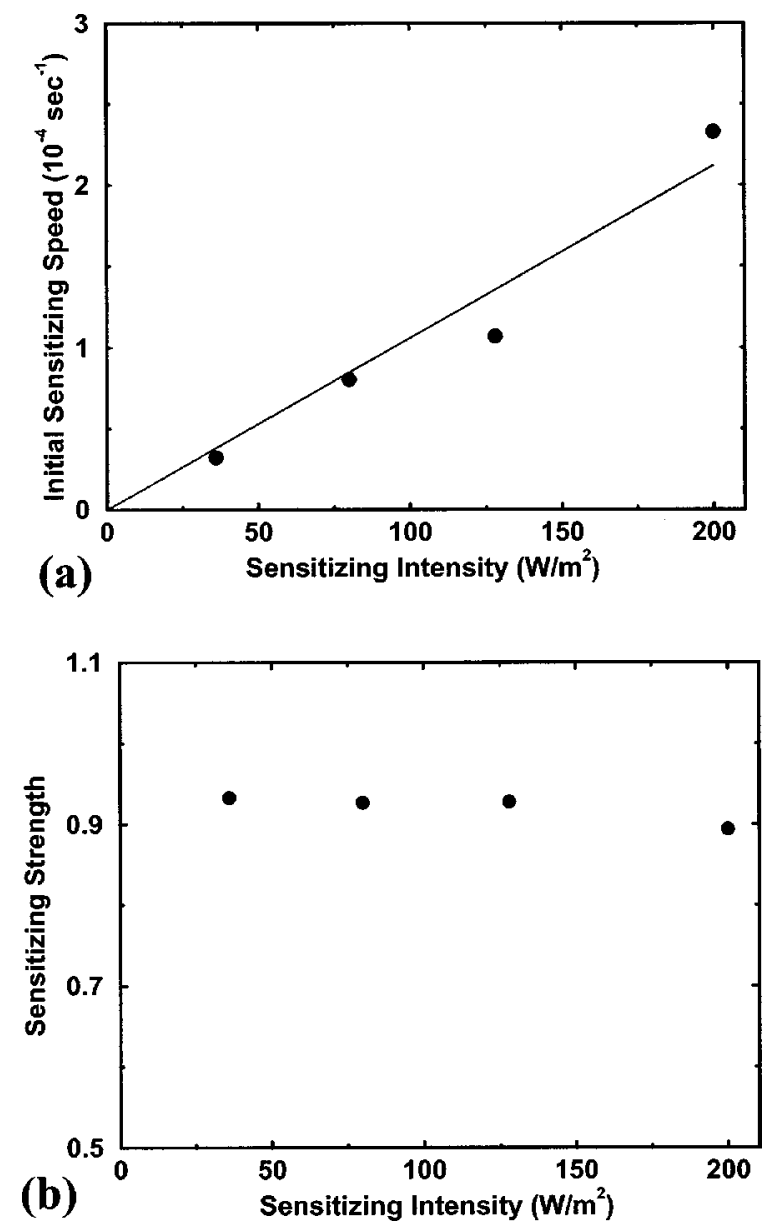

Fig. 7. Variation of (a) initial sensitization speed $\left.\left\{\left|\left[\mathrm{d}\left(I_{t} / I_{i}\right) / \mathrm{d} t\right]\right|_{t=0} \mid\right\} / I_{\mathrm{UV} 0}\right)$ and (b) sensitization strength (ratio of the final transmitted red power to the initial transmitted power) with sensitizing intensity $\left(I_{\mathrm{UV} 0}\right)$. Solid line, linear fit to the experimental data.

traps at $365 \mathrm{~nm}\left(\kappa_{\mathrm{Mn}, \mathrm{UV}}\right)$, which one can find by fitting the theoretical and experimental results. The final values of the parameters are listed in Table 1.

\section{Comparison with the Experimental Results}

In fitting the theoretical curves to the experimental results we try to get the best overall fit to the experimental sensitization, bleaching, and holographic recording and readout results. The simulation of the sensitization, bleaching, and holographic recording curves is based on the numerical solutions of the governing differential equations. To consider the absorption of UV light within the crystal, we divided the crystal into 50 thin portions with equal thickness. The intensity of the UV light was assumed to be constant in each portion. The corresponding equations were solved within each portion. We then calculated the overall response of the entire crystal by combining the relevant results (optical density in sensitization-bleaching and index change in holographic recording) of the different portions appropriately.

Figure 8 shows a comparison of the theoretical and experimental results for the best overall fit. The agreement between theory and experiment shown in Fig. 8 is good, especially for the beginning of the recording, satu- ration diffraction efficiency, and the final diffraction efficiency (after sufficient readout), which are the important features of the recording and readout curves used in the computation of sensitivity and $M / \#$. The final values of all parameters that result in the theoretical curves in Fig. 8 are summarized in Table 1 .

\section{Effect of Sensitizing and Recording Intensities}

Figure 9(a) shows the theoretical saturation diffraction efficiency as a function of the recording intensity $\left(I_{\mathrm{R} 0}\right)$ when the ratio of the recording intensity to sensitizing intensity is fixed $\left(I_{\mathrm{R} 0} / I_{\mathrm{UV} 0}=25\right)$. We calculate one of the curves in Fig. 9(a) by neglecting the absorption of the sensitizing beam $\left(\alpha_{\mathrm{UV}}=0\right)$, whereas the other curve is calculated with the absorption of the sensitizing beam included $\left(\alpha_{\mathrm{UV}}=9 \mathrm{~mm}^{-1}\right)$. Neglect of this absorption is an acceptable assumption only for thin crystals or for cases in which we are interested in local hologram strength.
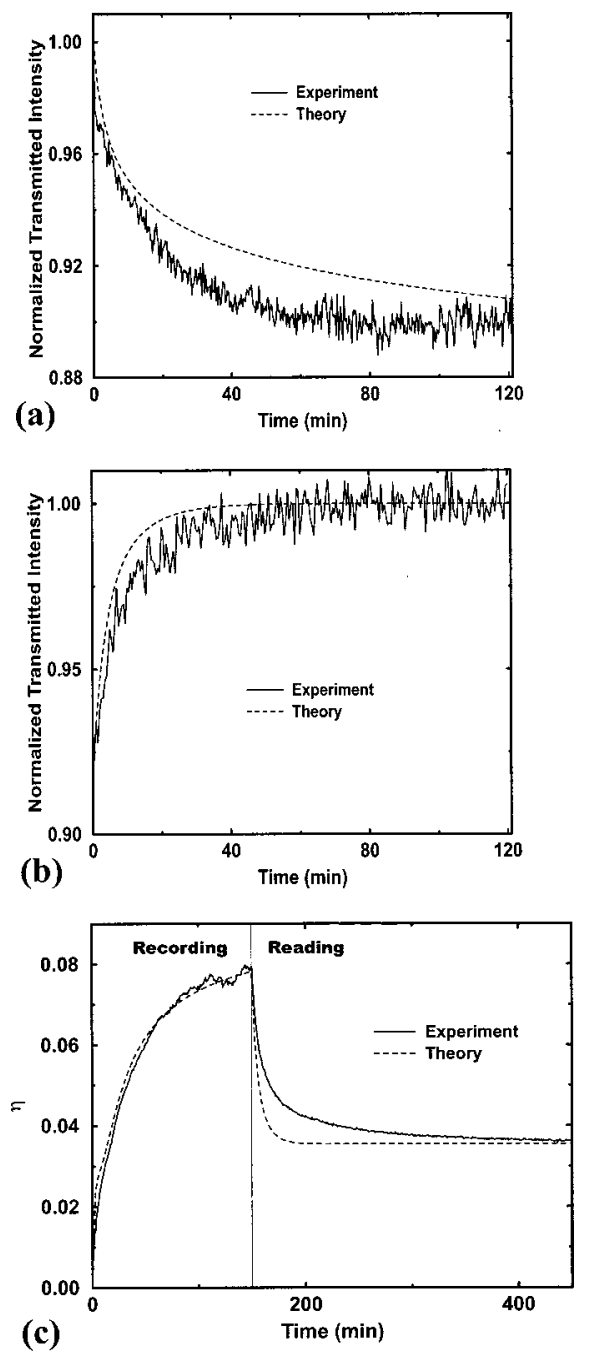

Fig. 8. Comparison of theory and experiment: (a) Sensitization by a $20-\mathrm{mW} / \mathrm{cm}^{2}$ homogeneous UV beam at $365 \mathrm{~nm}$ monitored by a weak red beam (wavelength, $633 \mathrm{~nm}$ ). (b) Bleaching of a sensitized crystal by a $300-\mathrm{mW} / \mathrm{cm}^{2}$ red beam. (c) Holographic recording by simultaneous presence of a UV beam (wavelength, $365 \mathrm{~nm}$; intensity, $20 \mathrm{~mW} / \mathrm{cm}^{2}$ ) and two red beams (wavelength, $633 \mathrm{~nm}$; intensity of each beam, $250 \mathrm{~mW} / \mathrm{cm}^{2}$; ordinary polarization) with subsequent readout achieved by one of the red recording beams only. 

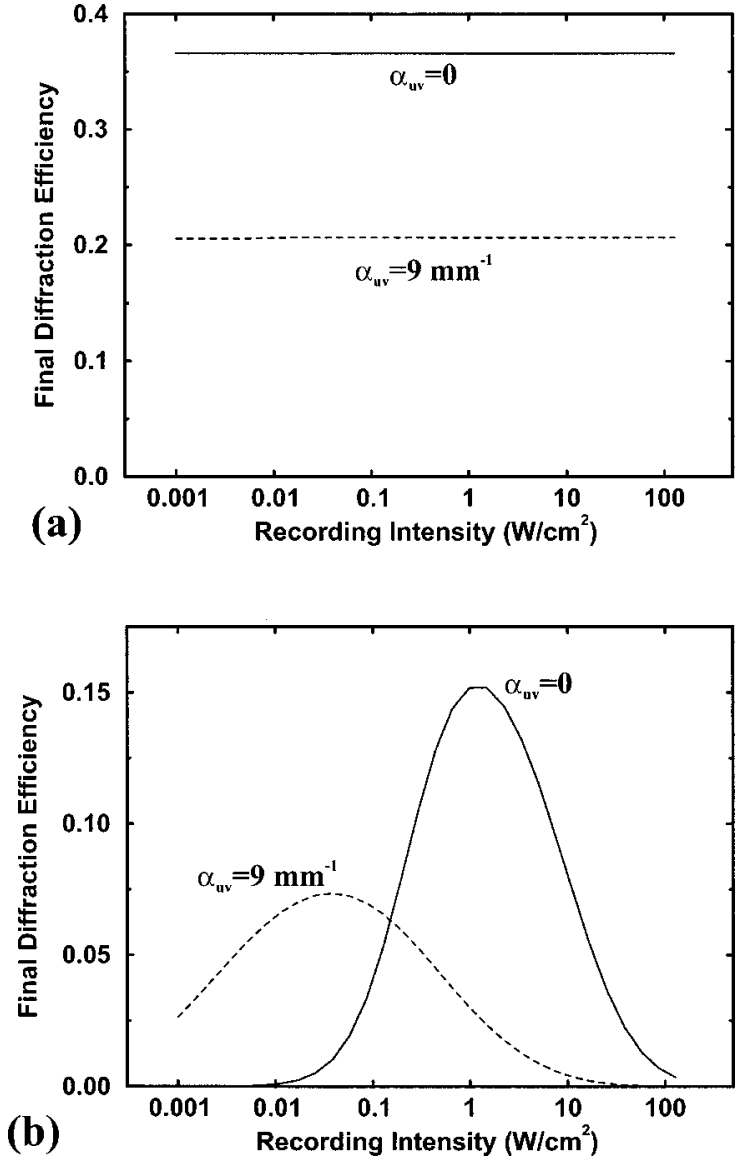

Fig. 9. Variation of the final persistent diffraction efficiency with recording intensity while (a) the ratio of the recording to sensitizing intensity is fixed $\left(I_{R 0} / I_{\mathrm{UV} 0}=25\right)$ and (b) the sensitizing intensity is fixed $\left(I_{\mathrm{UV} 0}=20 \mathrm{~mW} / \mathrm{cm}^{2}\right)$. The wavelength of the sensitizing beam in the calculations is $365 \mathrm{~nm} . \alpha_{\mathrm{UV}}=0$ (solid) and $\alpha_{\mathrm{UV}}=9 \mathrm{~mm}^{-1}$ (dashed) are the variation when the absorption of the UV light within the crystal is and is not neglected, respectively.

However, this rule does not apply to thick crystals because UV absorption cannot be neglected in such crystals. Typical absorption coefficients of the crystals that we used are nearly $9 \mathrm{~mm}^{-1}$ at $365 \mathrm{~nm}$. Figure $9(\mathrm{~b})$ shows the variation of the saturation diffraction efficiency with recording intensity $\left(I_{\mathrm{R} 0}\right)$ at constant sensitizing intensity $\left(I_{\mathrm{UV} 0}=20 \mathrm{~mW} / \mathrm{cm}^{2}\right)$. Figure 9 suggests that the final diffraction efficiency in two-center holographic recording is a function of the intensity ratio $\left(I_{\mathrm{R} 0} / I_{\mathrm{UV} 0}\right)$ only and not a function of the absolute intensities. This result can be intuitively understood when we recall that the sensitizing light populates and the recording light depopulates the $\mathrm{Fe}$ traps. The strengths of the processes caused by sensitizing and recording lights depend on sensitizing and recording intensities, respectively. Therefore, if we change the sensitizing and recording intensities while keeping their ratio constant, we will not change the relative roles of the processes involved in recording the hologram, and we will obtain the same saturation diffraction efficiency. Note that the holographic recording speed still depends on the absolute intensities, as stronger beams result in faster responses.

Figure 9(b) shows that the final diffraction efficiency depends more broadly on the recording intensity for higher UV absorption. This is so because the ratio of the recording and UV intensities $\left(I_{\mathrm{R} 0} / I_{\mathrm{UV} 0}\right)$ varies through the thickness of the crystal, as the absorption of the recording light is much weaker than that of the UV light. Therefore the best UV intensity that corresponds to the approximately fixed recording intensity cannot be provided for all points within the thickness of the crystal. If the UV intensity is high enough, there is a relatively narrow region within the crystal with optimum intensity ratio. When the UV intensity is increased, this narrow region moves away from the UV entrance edge. If we increase the UV intensity beyond some maximum value, there is no region within the crystal with optimum intensity ratio, as the UV intensity remains too high at all points within the crystal thickness. For UV intensities above that maximum value, the final diffraction efficiency decreases with increasing UV intensity.

Finally, it is important to note that there is no intensity threshold for two-center holographic recording, as shown in Fig. 9. We can record holograms with low recording and UV intensities and obtain large diffraction efficiencies if the intensity ratio is picked properly. This is a big advantage of two-center recording over two-step persistent holographic recording with small polarons in $\mathrm{LiNbO}_{3}:$ Fe crystals. ${ }^{21}$

\section{E. Importance of Sensitizing Light}

As was shown in Fig. 4, the presence of UV light during hologram formation is crucial for obtaining large diffraction efficiencies. In this subsection we explain the physical reason for this importance.

The electron recombination rates of $\mathrm{Mn}$ and Fe centers have the same order of magnitude. Therefore the probabilities of trapping a conduction-band electron at $\mathrm{Mn}$ and $\mathrm{Fe}$ sites are comparable. As a result, when an electron is excited from $\mathrm{Fe}$ to the conduction band, it will quickly end up in Mn centers after a few Fe retrapping cycles. An electron moves only a few nanometers in the conduction band before getting retrapped at either center as a result of the small mobility of electrons in $\mathrm{LiNbO}_{3}$. Therefore, if there is no UV illumination during recording, an electron moves only a few nanometers on the average before it is trapped in Mn centers, a distance much smaller than the grating period (usually $\sim 1 \mu \mathrm{m}$ ). Once an electron is trapped in $\mathrm{Mn}$, it can no longer be used for holographic recording. Having simultaneous UV illumination during recording makes the Mn electrons available for recording and increases the average distance by which an electron can move through multiple cycles of excitation, which results in successful recording of gratings in $\mathrm{Mn}$ to large saturation diffraction efficiencies.

In recording a hologram in the $\mathrm{LiNbO}_{3}: \mathrm{Fe}: \mathrm{Mn}$ crystal, the main source for moving electrons in the conduction band is the bulk photovoltaic effect $\left(j_{\mathrm{ph}}=\kappa_{\mathrm{Fe}, R} N_{\mathrm{Fe}}{ }^{-} I_{R}\right)$. At the beginning of recording, the electron concentration in the Fe traps $\left(N_{\mathrm{Fe}}{ }^{-}\right)$is uniform. Therefore the bulk photovoltaic effect is maximum at the peaks of the red light intensity $\left(I_{R}\right)$. Without the presence of UV light during recording, the recording light bleaches the Fe centers. This bleaching is faster in high light intensity regions, resulting in a $180^{\circ}$ phase difference between $N_{\mathrm{Fe}}{ }^{-}$ 
and $I_{R}$. Assuming sinusoidal variation with space, we can represent the first Fourier component of the bulk photovoltaic current as

$$
j_{\mathrm{ph} 1}=\kappa_{\mathrm{Fe}, R}\left(N_{\mathrm{Fe} 0}{ }^{-} I_{R 1}-N_{\mathrm{Fe} 1}{ }^{-} I_{R 0}\right),
$$

where we have assumed that

$$
\begin{aligned}
I_{R} & =I_{R 0}+I_{R 1} \exp (i K x), \\
N_{\mathrm{Fe}}{ }^{-} & =N_{\mathrm{Fe} 0}{ }^{-}-N_{\mathrm{Fe} 1}{ }^{-} \exp (i K x),
\end{aligned}
$$

with all parameters defined as above. Therefore, at some point in time, the bulk photovoltaic current at the peaks of the interference pattern becomes weaker than the bulk photovoltaic current at positions away from the peaks, reversing the prevailing charge-transfer direction and causing erasure. The peak in the recording curve of Fig. 4 (without UV light) corresponds to this reversal of direction.

The argument presented above is useful for an intuitive understanding of the processes. We can also use our theoretical model to explain the experimental measurements in Fig. 4. Figure 10 shows the concentration of filled Fe centers $\left(N_{\mathrm{Fe}}{ }^{-}\right)$and photovoltaic current at different times during hologram formation as a function of position $x$. In calculating the results shown in Figs. 10(a) and 10(b) we assumed that recording is performed by red only (without UV) light and by the simultaneous presence of UV and red light, respectively. In both cases we assumed that recording is done after presensitization of the crystal with UV light.
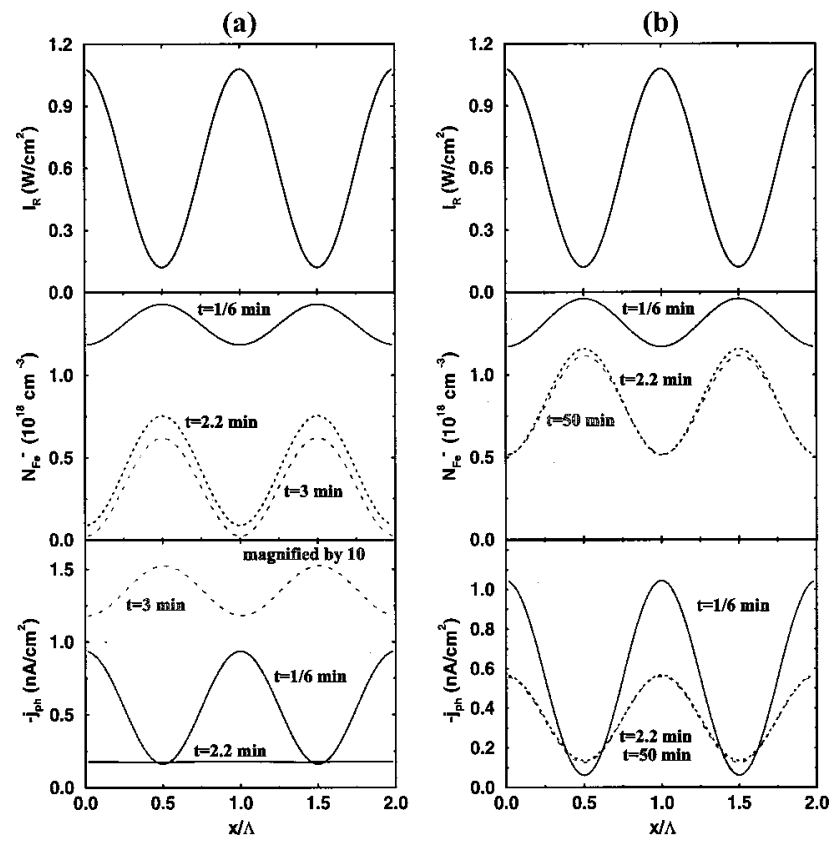

Fig. 10. Spatial variations of recording intensity $\left(I_{R}\right)$, electron concentration in Fe traps $\left(N_{\mathrm{Fe}}{ }^{-}\right)$, and bulk photovoltaic current $\left(j_{\mathrm{ph}}\right)$ over two grating periods $(\Lambda)$ at different times in a thin slice of the crystal during holographic recording. Recording is accomplished by two red beams (wavelength, $633 \mathrm{~nm}$; intensity of each beam, $300 \mathrm{~mW} / \mathrm{cm}^{2}$; ordinary polarization) (a) without UV illumination during recording, and (b) with simultaneous illumination with a UV beam (wavelength, $365 \mathrm{~nm}$; intensity, $20 \mathrm{~mW} / \mathrm{cm}^{2}$ ). In both cases it is assumed that the crystal was preilluminated by the UV beam for two hours before recording.
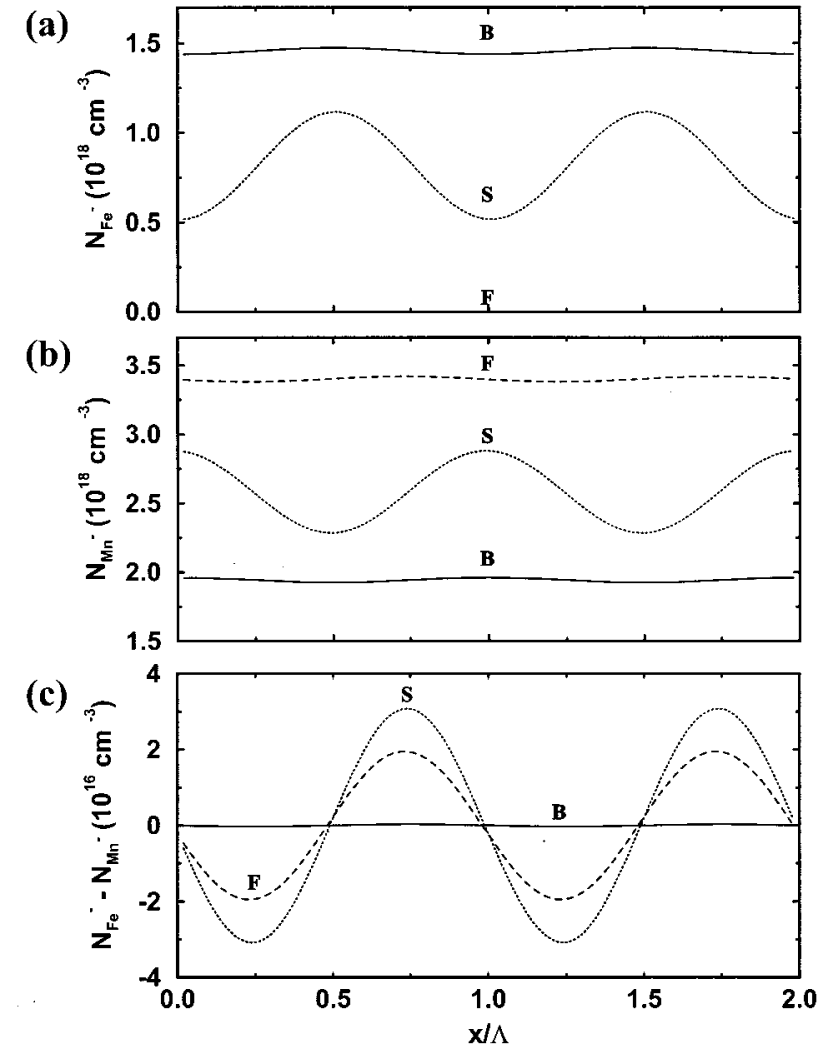

Fig. 11. Spatial variations of electron concentrations in (a) Fe $\left(N_{\mathrm{Fe}}{ }^{-}\right)$and (b) Mn traps $\left(N_{\mathrm{Mn}^{-}}{ }^{-}\right)$and (c) their sum $\left(N_{\mathrm{Fe}}{ }^{-}\right.$ $+N_{\mathrm{Mn}}{ }^{-}$) over two grating periods $(\Lambda)$ at different times (B, at the beginning of recording, $\mathrm{S}$, at saturation, and F, after sufficient readout) in a thin slice of the crystal during holographic recording. Recording is achieved by two red beams (wavelength, $633 \mathrm{~nm}$; intensity of each beam, $300 \mathrm{~mW} / \mathrm{cm}^{2}$; ordinary polarization) with simultaneous illumination with a UV beam (wavelength, $365 \mathrm{~nm}$; intensity, $20 \mathrm{~mW} / \mathrm{cm}^{2}$ ). Note that the spatial variation of $\mathrm{N}_{\mathrm{Fe}}{ }^{-}$has a $180^{\circ}$ phase shift from that of the recording intensity, as shown in Fig. 10(b).

Figure 10(a) shows clearly that the reversal in the direction of charge transfer is responsible for the fall of the diffraction efficiency when the UV light is not present during hologram formation. Furthermore, Fig. 10(b) shows that the presence of UV light during recording results in a nonzero steady-state electron concentration in the Fe traps. This is so because of continuous sensitization (electron transfer from Mn traps to Fe traps) by UV light. As a result, reversal in the charge-transfer direction in the conduction band does not occur, and a strong hologram can be recorded.

It is important to note that the space-charge pattern resides in both centers because the recombination rate of the electrons from the conduction band for the Fe and Mn centers are similar each other. Therefore, modulated $\mathrm{Fe}^{2+}$ and $\mathrm{Mn}^{2+}$ gratings are formed during recording. The sum of these gratings produces the space-charge field. Figure 11 shows the concentration of electrons in Fe and Mn traps at different times during recording with the simultaneous presence of UV light. B, S, and F in Fig. 11 refer to beginning of recording, saturation, and the final part of the process (after sufficient readout), respectively. The $180^{\circ}$ phase difference between the two gratings results from the fact that, in bright red regions, 
electrons are transferred from Fe centers to Mn centers at maximum rate. Therefore the $\mathrm{Mn}^{2+}$ grating is maximum in the brighter red regions and minimum in the darker areas. The situation for the $\mathrm{Fe}^{2+}$ grating is the reverse, introducing a $180^{\circ}$ phase difference between the two sinusoidal patterns. The real phase difference is a bit smaller than $180^{\circ}$ owing to the movement of electrons in the conduction band before they are retrapped. During readout with red light only, electrons in the Fe centers move in the reverse direction, resulting in partial erasure of the hologram. The hologram is not completely erased because the electrons can move only a short distance before they are trapped in the Mn centers. After sufficient readout, all $\mathrm{Fe}$ traps will become empty, and the final grating will remain recorded in the Mn traps.

\section{OPTIMIZATION OF TWO-CENTER RECORDING}

In this section we focus on the effects of individual design parameters (i.e., $\mathrm{Fe}$ and $\mathrm{Mn}$ concentrations, initial electron concentrations in the traps, recording and sensitizing intensities and wavelengths, etc.) on the dynamic range of two-center holographic recording systems. A convenient measure of the dynamic range is $M / \# .^{35}$ An approximate measure of $M / \#$ in a two-center holographic recording scheme is the square root of the final persistent diffraction efficiency. We consider the effect of each of the design parameters on this measure when all other design parameters are fixed. The effects of recording and sensitizing intensities were considered in Section 4.

\section{A. Effect of Fe Concentration}

Figure 12(a) shows the theoretical variation of the approximate $M / \#$ with $\mathrm{Fe}$ concentration while the $\mathrm{Mn}$ concentration is fixed at $N_{\mathrm{Mn}}=3.8 \times 10^{24} \mathrm{~m}^{-3}$ (corresponding to 0.01 wt. \% $\mathrm{MnO}$ doping). In this calculation we have assumed that all Fe traps are initially empty and that $90 \%$ of the $\mathrm{Mn}$ traps are occupied by electrons $\left(N_{A}\right.$ $\left.=3.4 \times 10^{24} \mathrm{~m}^{-3}\right)$. As Fig. 12(a) shows, stronger holograms and larger $M / \#$ are obtained at higher Fe concentrations. The variation in Fig. 12(a) can be understood from the energy-band diagram in Fig. 1. Without any Fe traps, we cannot record any holograms, as red light cannot excite electrons from $\mathrm{Mn}$ traps to the conduction band. By increasing the concentration of the Fe traps (keeping them all initially empty), we increase the probability of trapping electrons from the conduction band by Fe traps, which increases the concentration of electrons in the Fe traps during recording. Therefore, stronger holograms can be recorded and a larger $M / \#$ obtained. In the calculation shown in Fig. 12(a) we considered only the practical $\mathrm{Fe}$ concentration in $\mathrm{LiNbO}_{3}$ (up to 0.15 wt. \% $\mathrm{Fe}_{2} \mathrm{O}_{3}$ ). However, strong absorption of the recording beams may also limit the highest useful Fe concentration.

Figure 12(b) shows the result of holographic recording and readout experiments performed with two different $\mathrm{LiNbO}_{3}: \mathrm{Fe}: \mathrm{Mn}$ crystals with the same $\mathrm{Mn}$ concentration and thickness but different Fe concentrations. The crystals were also annealed together. Recording and readout are accomplished with the same system parameters (intensities, grating period, etc.) in both crystals. The ex-
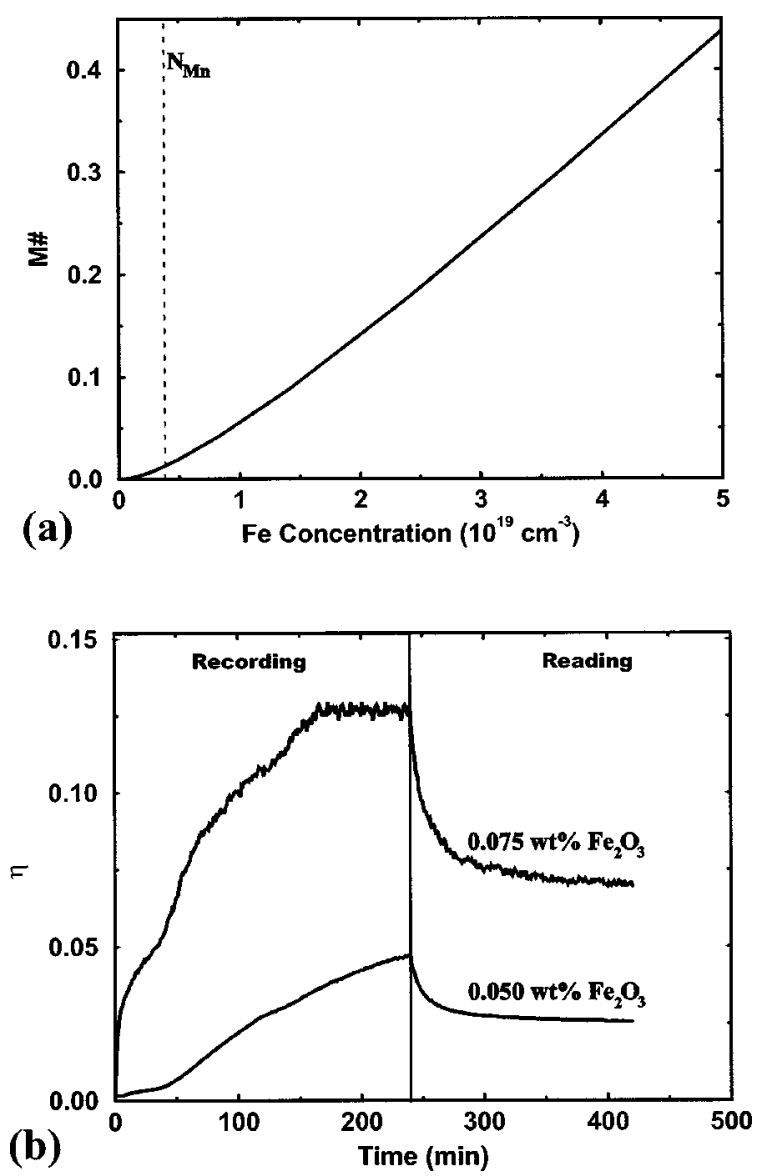

Fig. 12. Effect of Fe concentration on two-center holographic recording in $\mathrm{LiNbO}_{3}: \mathrm{Fe}: \mathrm{Mn}$ crystals. (a) Theoretical variation of the final hologram strength (approximate $M / \#$ ) with Fe concentration while the $\mathrm{Mn}$ concentration is fixed at $3.8 \times 10^{18} \mathrm{~cm}^{-3}$ (equivalent to $0.01 \mathrm{wt} \% \% \mathrm{MnO}$ ). (b) Recording and readout curves for two $\mathrm{LiNbO}_{3}: \mathrm{Fe}: \mathrm{Mn}$ crystals, each doped with $0.01 \mathrm{wt}$. \% $\mathrm{MnO}$. The Fe doping level for each crystal is shown. Recording is accomplished by a UV beam (wavelength, $404 \mathrm{~nm}$; intensity, 4 $\mathrm{mW} / \mathrm{cm}^{2}$ ) and two red beams (wavelength, $633 \mathrm{~nm}$; intensity of each beam, $300 \mathrm{~mW} / \mathrm{cm}^{2}$; ordinary polarization). Readout uses one of the red recording beams only.

perimental parameters are given in the figure caption. As Fig. 12(b) shows, the crystal with 50\% more Fe traps has approximately $50 \%$ larger $M / \#$, as suggested by Fig. 12(a).

The theoretical and experimental results shown in Fig. 12 suggest that, in designing a material for two-center holographic recording, we should choose the highest practical doping level for the shallower traps (i.e., $\mathrm{Fe}$ in $\left.\mathrm{LiNbO}_{3}: \mathrm{Fe}: \mathrm{Mn}\right)$.

\section{B. Effect of Mn Concentration}

Figure 13(a) shows the theoretical variation of the approximate $M / \#$ with $\mathrm{Mn}$ concentration while the Fe concentration is fixed at $N_{\mathrm{Fe}}=2.5 \times 10^{25} \mathrm{~m}^{-3}$ (corresponding to 0.075 wt. $\% \mathrm{Fe}_{2} \mathrm{O}_{3}$ doping). In this calculation we assumed that all Fe traps are initially empty and that $90 \%$ of the Mn traps are occupied by electrons. All other parameters are kept constant (as stated in the caption). As Fig. 13(a) shows, there is an optimum Mn concentration. Without any Mn traps, we cannot record a hologram because all Fe traps are initially empty. Therefore, 
we have $M / \#=0$ at zero Mn concentration. By increasing the Mn concentration from zero (while keeping $90 \%$ of them filled with electrons), we increase the number of electrons available for sensitization. Therefore we get a larger electron excitation rate from Mn traps to the conduction band (rate 1 in Fig. 1). However, as $10 \%$ of the Mn traps are empty, we also increase the rate of electron trapping at Mn centers (rate 3 in Fig. 1) by increasing the Mn concentration. This militates against sensitization (or electron transfer to Fe traps). Therefore when we increase the Mn concentration we have two competing effects that explain the occurrence of a maximum in the variation of the $M / \#$ with the concentration of the $\mathrm{Mn}$ traps. For the intensity ratio chosen in this calculation, the optimum occurs when the Mn concentration is approximately $10 \%$ of the Fe concentration.

Figure 13(b) shows the results of holographic recording and readout experiments performed with three different $\mathrm{LiNbO}_{3}: \mathrm{Fe}: \mathrm{Mn}$ crystals with the same Fe concentration
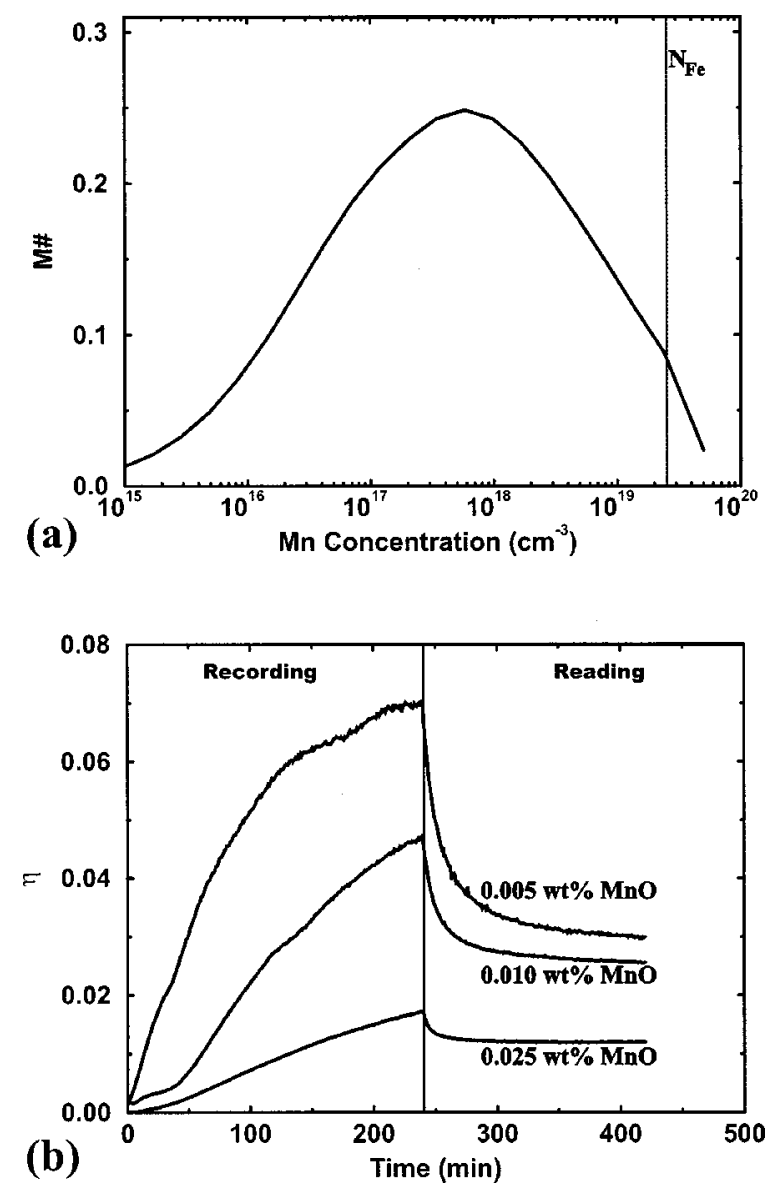

Fig. 13. Effect of Mn concentration on two-center holographic recording in $\mathrm{LiNbO}_{3}: \mathrm{Fe}: \mathrm{Mn}$ crystals. (a) Theoretical variation of the final hologram strength (approximate $M / \#$ ) with Mn concentration while the Fe concentration is fixed at $2.5 \times 10^{19} \mathrm{~cm}^{-3}$ (equivalent to 0.075 wt. $\% \mathrm{Fe}_{2} \mathrm{O}_{3}$ ). In the simulation it is assumed that all Fe traps are empty and that $90 \%$ of the Mn traps are filled with electrons. (b) Recording and readout curves for three $\mathrm{LiNbO}_{3}: \mathrm{Fe}: \mathrm{Mn}$ crystals, each doped with 0.05 wt. $\% \mathrm{Fe}_{2} \mathrm{O}_{3}$. The Mn doping level for each crystal is shown. Recording is achieved by a UV beam (wavelength, $404 \mathrm{~nm}$; intensity, 4 $\mathrm{mW} / \mathrm{cm}^{2}$ ) and two red beams (wavelength, $633 \mathrm{~nm}$; intensity of each beam, $300 \mathrm{~mW} / \mathrm{cm}^{2}$; ordinary polarization). Readout uses one of the red recording beams only. and thickness but different Mn concentrations. The crystals were also annealed together such that they have similar initial electron concentrations in the Mn traps while all Fe traps are initially empty. Recording and readout are accomplished with the same system parameters (intensities, grating period, etc.) in both crystals. The experimental parameters are given in the caption. As Fig. 13(b) shows, the crystal whose Mn concentration is approximately $10 \%$ of its Fe concentration has the largest persistent diffraction efficiency (and therefore the largest $M / \#)$. The persistent diffraction efficiency is smaller for crystals with higher Mn concentrations. The experimental results confirm the latter part (after the maximum) of Fig. 13(a). If we believe that no hologram can be recorded $(M / \#=0)$ without any $\mathrm{Mn}$ traps, we can conclude from the experimental results that there is a $\mathrm{Mn}$ concentration that results in maximum $M / \#$.

The theoretical and experimental results shown in Fig. 13 suggest that, in designing a material for two-center holographic recording, we should choose the optimum doping level for the deeper traps (i.e., $\mathrm{Mn}$ in $\mathrm{LiNbO}_{3}: \mathrm{Fe}: \mathrm{Mn}$ ). As a rule of thumb, the optimum for any $\mathrm{LiNbO}_{3}: \mathrm{Fe}: \mathrm{Mn}$ crystal occurs when the Mn concentration is approximately $10 \%$ of the Fe concentration. Although this result is obtained for a specific crystal and a specific intensity ratio, we expect it to be a good starting point in the design of a $\mathrm{LiNbO}_{3}: \mathrm{Fe}: \mathrm{Mn}$ crystal for two-center holographic recording. For any other material and or dopant, there is always an optimum concentration for the deeper traps that can be found by the method that we have described.

\section{Effect of Annealing}

Because holographic recording is achieved by spatial rearrangement of the electrons in the Fe and Mn traps, the initial electron concentration in the traps has an important effect on the holographic recording performance. The electron concentration in the traps can be varied by annealing (or oxidation-reduction $)^{24}$ of the crystal. Because Mn traps are deeper in the bandgap than Fe traps, electrons fill the Mn traps before Fe traps when the crystal is reduced. For persistent holographic recording, it is essential that at the end of the annealing process all $\mathrm{Fe}$ traps be empty and that only a portion of the Mn traps be filled. Figure 14(a) shows the theoretical variation of the approximate $M / \#$ with the portion of filled Mn traps, whereas the $\mathrm{Mn}$ and Fe concentrations are fixed at $N_{\mathrm{Mn}}$ $=3.81 \times 10^{24} \mathrm{~m}^{-3}$ (corresponding to $0.01 \mathrm{wt} \% \mathrm{MnO}$ doping) and $N_{\mathrm{Fe}}=2.5 \times 10^{25} \mathrm{~m}^{-3}$ (corresponding to 0.075 wt. $\% \mathrm{Fe}_{2} \mathrm{O}_{3}$ doping), respectively. In this calculation we assumed that all Fe traps are initially empty, as required to obtain persistence. As Fig. 14(a) shows, there is an optimum annealing (or oxidation-reduction) state for the crystal that results in the best $M / \#$. For the crystal with specifications given above, the optimum annealing state is approximately $97 \%-98 \%$ of the Mn traps filled with all the Fe traps empty. The optimum value is a sensitive function of $\mathrm{Mn}$ and Fe concentrations. For small variations of these concentrations from those of the given crystal, we expect the optimum annealing state to be somewhere between $95 \%$ and $100 \%$ of the Mn traps filled with all Fe traps empty. 

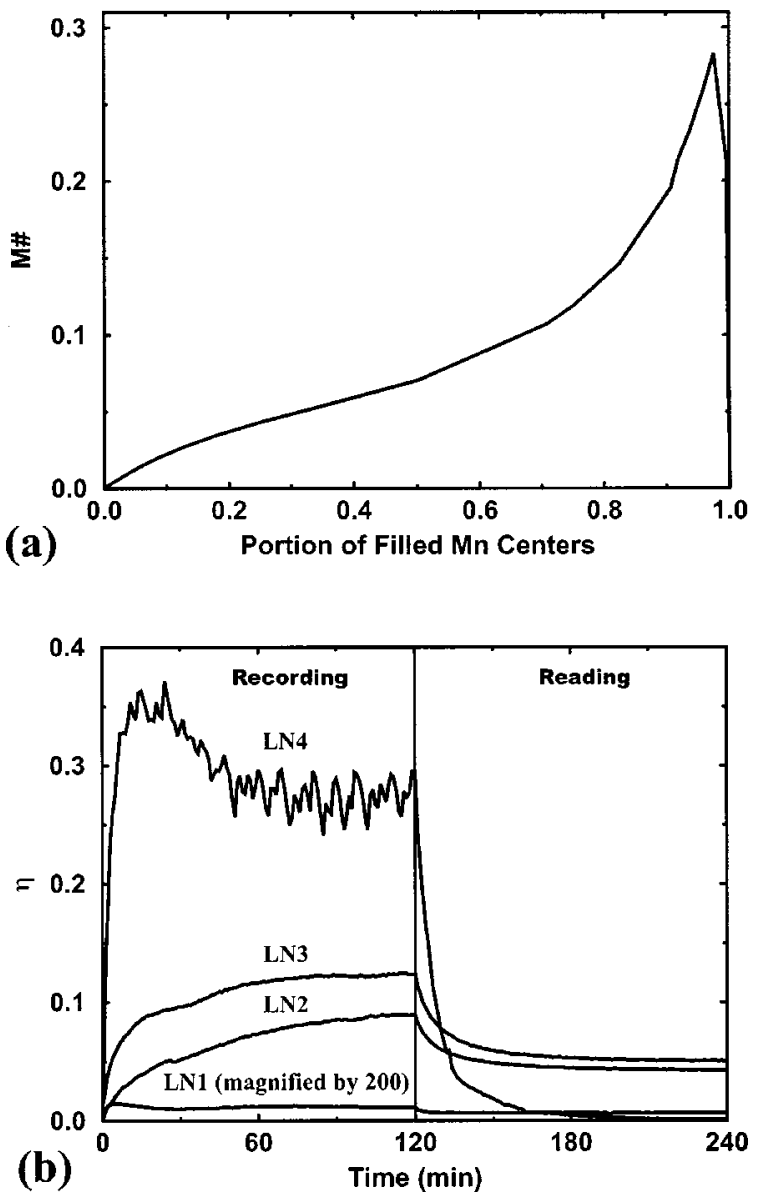

Fig. 14. Effect of annealing on two-center holographic recording in $\mathrm{LiNbO}_{3}: \mathrm{Fe}: \mathrm{Mn}$ crystals. (a) Theoretical variation of the final hologram strength (approximate $M / \#$ ) with the portion of filled $\mathrm{Mn}$ traps while the $\mathrm{Fe}$ and $\mathrm{Mn}$ concentrations are fixed at $2.5 \times 10^{19} \mathrm{~cm}^{-3}$ (equivalent to 0.075 wt. $\% \mathrm{Fe}_{2} \mathrm{O}_{3}$ ) and $3.8 \times 10^{18}$ $\mathrm{cm}^{-3}$ (equivalent to $0.01 \mathrm{wt}$ \% $\mathrm{MnO}$ ), respectively. (b) Recording and readout curves for four $\mathrm{LiNbO}_{3}: \mathrm{Fe}: \mathrm{Mn}$ crystals, each doped with 0.075 wt. $\% \mathrm{Fe}_{2} \mathrm{O}_{3}$ and 0.01 wt. $\% \mathrm{MnO}$. The annealing is achieved differently for different crystals (as specified in the text). Recording is performed by a UV beam (wavelength, 365 $\mathrm{nm}$; intensity, $20 \mathrm{~mW} / \mathrm{cm}^{2}$ ) and two red beams (wavelength, 633 $\mathrm{nm}$; intensity of each beam, $300 \mathrm{~mW} / \mathrm{cm}^{2}$; ordinary polarization). Readout uses one of the red recording beams only.

To check the theoretical result and to investigate the effect of the oxidation-reduction state of the crystal, we performed experiments with four $x$-cut congruent $\mathrm{LiNbO}_{3}$ crystals doped with 0.075 wt. $\% \mathrm{Fe}_{2} \mathrm{O}_{3}$ and with 0.01 wt. \% $\mathrm{MnO}$. The crystals were all from the same boule. The samples were strongly oxidized (sample LN1), oxidized (LN2), weakly oxidized (LN3), and strongly reduced (LN4) by annealing at temperatures of $700-1000^{\circ} \mathrm{C}$ in oxygen or argon atmospheres for different times. Sample LN1 was $2.9 \mathrm{~mm}$ thick, and all others were $0.85 \mathrm{~mm}$ thick. The absorption spectra of crystals LN1, LN3, and LN4 are shown in Fig. 15. The absorption spectrum of LN2 is so close to that of LN3 that, to avoid confusion, we do not show it. Almost all traps in the highly oxidized crystal are empty, resulting in a limited amount of absorption above $420 \mathrm{~nm}$. The absorption below this wavelength comes from band-to-band absorption of $\mathrm{LiNbO}_{3}$, electron transfer from the valance band to $\mathrm{Fe}$ traps (hole generation), ${ }^{36}$ and possibly some excitation of the few remaining electrons in Mn traps. As we reduce the oxidized sample, more Mn traps become occupied by electrons, resulting in stronger absorption above $420 \mathrm{~nm}$ (crystals LN2 and LN3). As we continue to reduce the sample, we reach a point at which all Mn traps are occupied by electrons and start to fill Fe traps with electrons. This causes an absorption band to appear at $\sim 477 \mathrm{~nm}$. The absorption in this band becomes stronger as we continue reducing the sample. The behavior observed in Fig. 15 implies that LN1 has hardly any electrons in either trap, LN2 and LN3 have partially filled Mn traps and empty Fe traps, and LN4 has completely filled Mn traps and partially filled Fe traps. No hologram can be recorded in LN1 because of the lack of electrons. Strong holograms can initially be recorded in LN4. However, the readout light causes electron transfer from $\mathrm{Fe}$ traps to Mn traps (via the conduction band) to fill all Mn traps during readout. The remaining hologram is in the Fe traps and is erased by further readout. During readout, therefore, we expect to find poor persistence when we use either LN1 or LN4. However, LN2 and LN3 are appropriate for persistent holographic recording, in complete agreement with experimental recording and readout curves for the four crystals shown in Fig. 14(b). A moredetailed explanation of the results shown in Fig. 14 can be found in Ref. 37.

To summarize, experimental results confirm the theoretical result that there is an optimum oxidationreduction state for a doubly doped $\mathrm{LiNbO}_{3}$ crystal that results in the desired performance. This optimum depends on the doping levels of the shallower $(\mathrm{Fe})$ and deeper $(\mathrm{Mn})$ traps and on the intensities of the sensitizing (UV) and recording (red) beams. Figure 14(a) shows that, for the crystal used in these experiments, the optimum oxidation-reduction state that results in the best $M / \#$ occurs when $\sim 97 \%-98 \%$ of the Mn traps are filled with electrons. This is close to the oxidation-reduction state of LN3. Once again, although this result is obtained for this specific crystal and a specific intensity ratio, we expect it to be a good starting point in the design of any $\mathrm{LiNbO}_{3}: \mathrm{Fe}: \mathrm{Mn}$ crystal for two-center holographic recording. For any other material or dopant, the optimum annealing occurs when all the shallower traps are empty and a good portion of the deeper traps are filled.

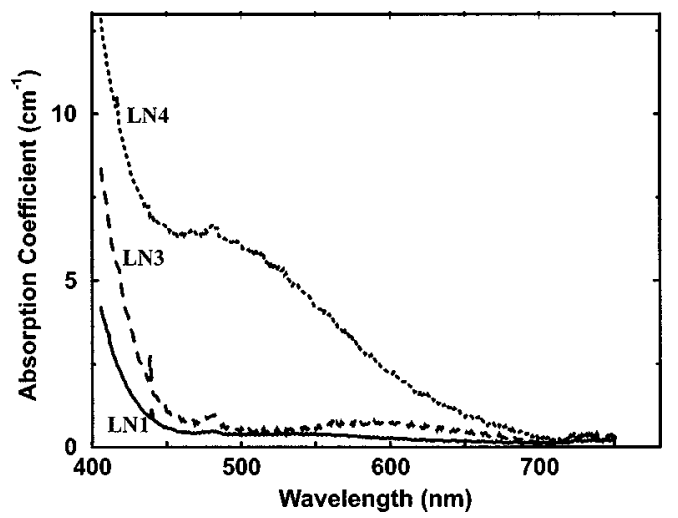

Fig. 15. Absorption spectra of three $\mathrm{LiNbO}_{3}: \mathrm{Fe}: \mathrm{Mn}$ crystals. The crystals are from the same boule, but they are annealed differently. 


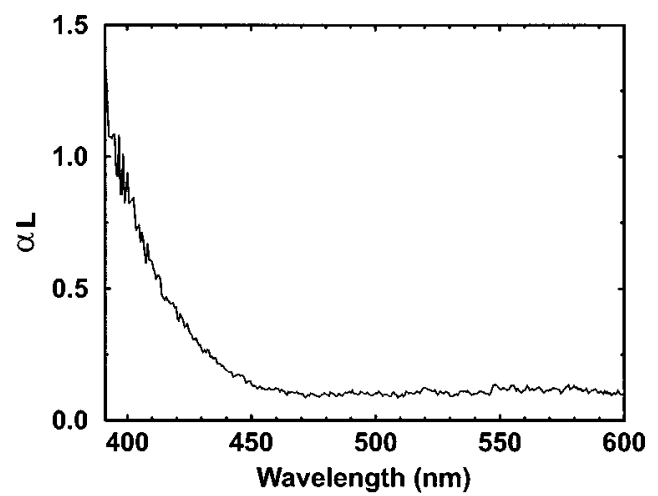

Fig. 16. Absorption spectrum of a typical $\mathrm{LiNbO}_{3}: \mathrm{Mn}$ crystal.
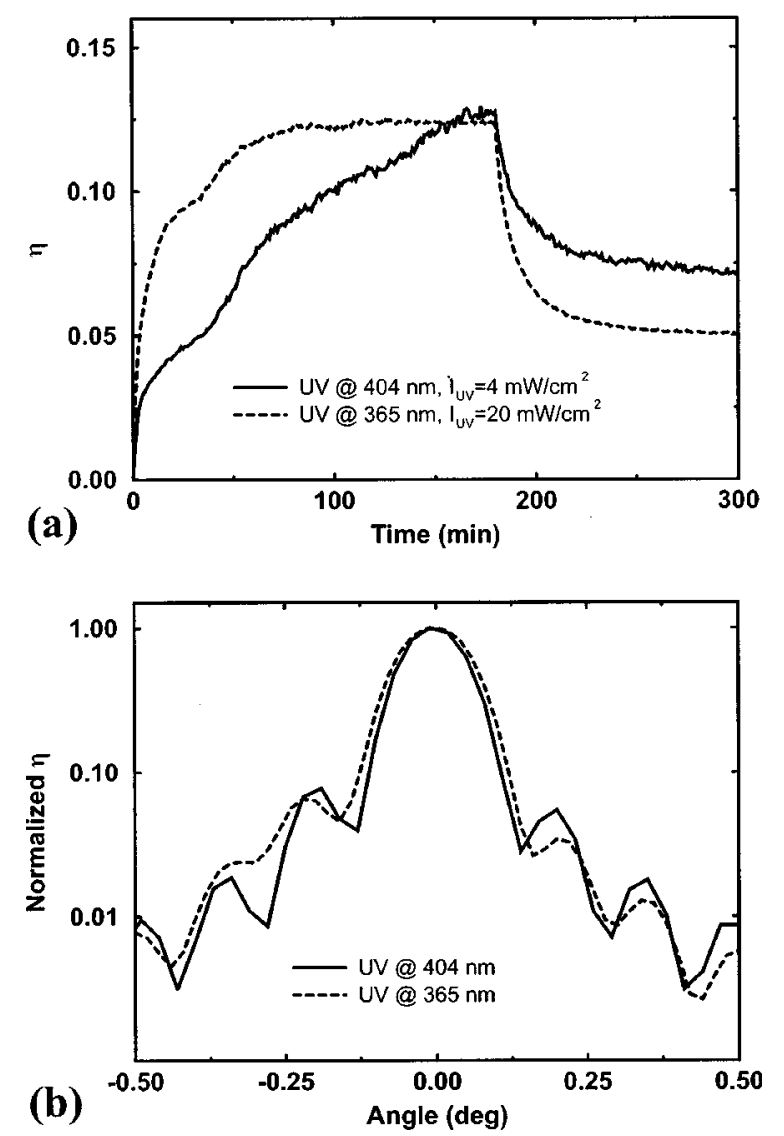

Fig. 17. Effect of sensitizing wavelength on two-center holographic recording in $\mathrm{LiNbO}_{3}: \mathrm{Fe}: \mathrm{Mn}$ crystals. (a) Recording and readout curves for a 0.85 -mm-thick $\mathrm{LiNbO}_{3}: \mathrm{Fe}: \mathrm{Mn}$ crystal doped with 0.075 wt. $\% \mathrm{Fe}_{2} \mathrm{O}_{3}$ and 0.01 wt. $\% \mathrm{MnO}$ with two different UV wavelengths. Recording is accomplished by a UV beam (wavelength and intensity in each case are specified) and two red beams (wavelength, $633 \mathrm{~nm}$; intensity of each beam, 300 $\mathrm{mW} / \mathrm{cm}^{2}$; ordinary polarization). Readout uses one of the red recording beams only. (b) Selectivity curves of two holograms recorded by the same two red beams and one UV beam with different wavelengths.

\section{Effect of Sensitizing Wavelength}

The maximum useful crystal thickness in two-center holographic recording depends on how deeply the sensitizing beam can penetrate the crystal. Therefore, large UV absorption results in a severe limitation in the maximum useful crystal thickness. The UV absorption coefficient of the $\mathrm{LiNbO}_{3}: \mathrm{Fe}: \mathrm{Mn}$ crystals that we used in the previous experiments was $9 \mathrm{~mm}^{-1}$ at $365 \mathrm{~nm}$. A large part of this absorption comes from band-to-band absorption that is not useful for sensitization.

To avoid the unwanted extra absorption of the sensitizing beam, we can increase the sensitizing wavelength. Higher sensitizing wavelengths result in weaker band-toband absorption and therefore in larger useful thickness of the material. The longer sensitizing wavelength, however, results in weaker excitation of the Mn traps. Figure 16 shows the absorption spectrum of a $\mathrm{LiNbO}_{3}$ crystal doped with Mn only. It is evident from Fig. 16 that we should not use sensitizing wavelengths above approximately $420 \mathrm{~nm}$. A practical wavelength below this limit is $404 \mathrm{~nm}$, which is available from a mercury lamp. Figure 17(a) shows the experimental recording and readout curves for a $\mathrm{LiNbO}_{3}: \mathrm{Fe}: \mathrm{Mn}$ crystal with sensitizing wavelength of 365 and $404 \mathrm{~nm}$. As Fig. 17(a) shows, using 404-nm sensitizing light results in larger persistent diffraction efficiency (and $M / \#$ ), even with five-times-lower sensitizing intensity. The recording speed for 365-nm sensitizing light is greater only because of the five-timeslarger sensitizing intensity at this wavelength. Figure 17(b) shows the angular selectivity curves (variation of the persistent diffraction efficiency with the angle of the reference beam) of a plane-wave hologram recorded in a 0.85-nm-thick $\mathrm{LiNbO}_{3}: \mathrm{Fe}: \mathrm{Mn}$ crystal with sensitizing wavelengths of 365 and $404 \mathrm{~nm}$. The hologram recorded with 404-nm sensitizing light is more selective, suggesting that the effective (or useful) crystal thickness is larger when we use 404-nm light for sensitization.

In general, to obtain the best performance in twocenter holographic recording we must choose a sensitizing wavelength that is long enough to prevent unwanted absorptions (band-to-band, etc.) and short enough to result in efficient sensitization from the deep traps. For $\mathrm{LiNbO}_{3}: \mathrm{Fe}: \mathrm{Mn}$ crystals, the best wavelength is in the 400-410-nm range.

\section{DISCUSSION}

From Fig. 17(a) we can obtain $M / \# \simeq 0.27$ by using 404-nm light for sensitization. We can improve $M / \#$ by nearly a factor of 3 by using extraordinary (in-plane) polarization for recording and readout beams because of the larger electro-optic coefficient of $\mathrm{LiNbO}_{3}$ for extraordinary polarization. Therefore we expect to have $M / \# \simeq 1$ for a 1-mm-thick crystal. In a practical system we would typically like to have a 1-cm-thick crystal. In the absence of absorption, $M / \#$ scales linearly with the crystal thickness, suggesting that $M / \# \simeq 10$ for a 1 -cm-thick sample. In normal holographic recording, the deviation in the linearity of $M / \#$ with thickness is not large, as the absorption of the recording beams can be adjusted by annealing. However, the absorption of the sensitizing beam in a twocenter holographic recording is typically much greater than that of the recording beams. This large absorption reduces the effective thickness of the crystal. Therefore we cannot obtain larger $M / \#$ by simply using thicker crystals. To get an idea of the largest useful thickness of a $\mathrm{LiNbO}_{3}: \mathrm{Fe}: \mathrm{Mn}$ in two-center holographic recording, we used the theoretical model to calculate the variation of 


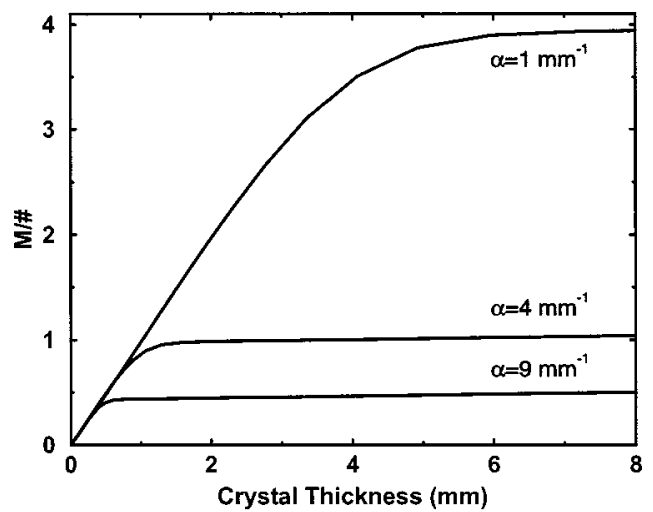

Fig. 18. Variation of the approximate $M / \#$ with crystal thickness in two-center holographic recording for different absorption coefficients of the sensitizing beam (intensity, $20 \mathrm{~mW} / \mathrm{cm}^{2}$ ). In this calculation we assumed that recording is achieved by the simultaneous presence of the sensitizing beam and two red beams (wavelength, $633 \mathrm{~nm}$; intensity of each beam, $300 \mathrm{~mW} / \mathrm{cm}^{2}$; ordinary polarizaton).

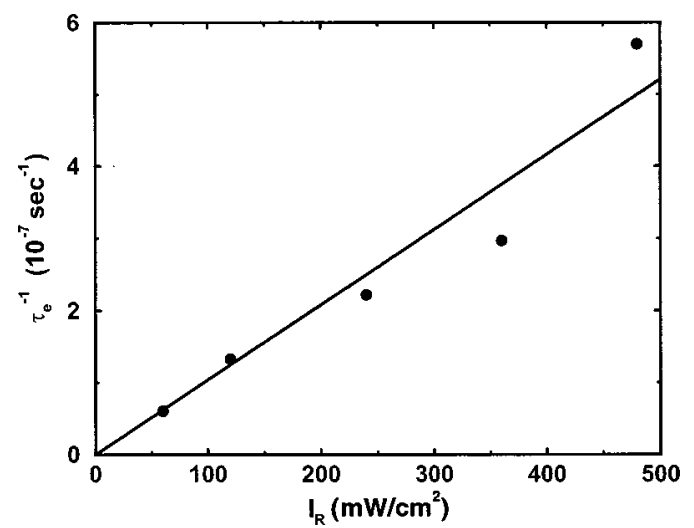

Fig. 19. Variation of erasure speed $\left(1 / \tau_{e}\right)$ with the intensity of the red reading beam $\left(I_{R}\right)$ in two-center holographic recording.

$M / \#$ with crystal thickness for different UV absorption coefficients $\left(\alpha_{\mathrm{UV}}\right)$. Figure 18 shows the results: the largest usable crystal thickness for $\alpha_{\mathrm{UV}}=9 \mathrm{~mm}^{-1}$ is $0.5 \mathrm{~mm}$. This is the case when the UV wavelength is $365 \mathrm{~nm}$. However, for $\alpha_{\mathrm{UV}}=1 \mathrm{~mm}^{-1}$ (for UV wavelength $404 \mathrm{~nm}$ ) we can use $4-5-\mathrm{mm}$-thick crystals. We can use 1 -cmthick samples if we use two sensitizing beams from the two opposite sides of the crystal. In this case, each beam sensitizes $5 \mathrm{~mm}$ of the crystal effectively, and therefore the entire crystal thickness is used for holographic recording. As a result, we expect $M / \#$ near 10 for a 1 -cm-thick crystal in the transmission geometry with extraordinary polarization.

Another important property for any holographic recording system is persistence (nondestructive readout). Twocenter holographic recording with red light has excellent persistence, as shown in Fig. 4. One measure of persistence is the erasure time constant during readout with some prespecified reading intensity. Figure 19 shows the variation of the erasure speed (the inverse of the erasure time constant) of the final hologram with the intensity of the reading red beam. To obtain the experimental data depicted in Fig. 19, first we recorded a hologram with the simultaneous presence of two red beams (wavelength, 633 $\mathrm{nm}$; intensity of each beam, $300 \mathrm{~mW} / \mathrm{cm}^{2}$; ordinary polar- ization) and one sensitizing beam (wavelength, $404 \mathrm{~nm}$; intensity, $4 \mathrm{~mW} / \mathrm{cm}^{2}$ ). Then we read out the hologram for $24 \mathrm{~h}$ with one of the red beams to make sure that almost all the electrons in Fe traps are transferred to $\mathrm{Mn}$ traps. Finally, we read out the hologram with one red beam with different intensities for at least $10 \mathrm{~h}$ in each case. We then calculate the erasure time constant $\left(\tau_{e}\right)$ by fitting the erasure curve to a monoexponential function such as

$$
\sqrt{\eta}=A \exp \left(-t / \tau_{e}\right)
$$

The deviation of the data from a linear curve is due partly to error in measuring the erasure time constant (because of the long duration of the erasure process) and partly to the fact that we have two traps instead of one. In other words, erasure from the Mn traps is achieved by redistribution of electrons among the Mn traps via the conduction band either without intermediate trapping at the Fe centers or with intermediate trapping at the $\mathrm{Fe}$ centers, and the corresponding erasure dynamics of these two erasure pathways are different.

Here we have used the saturation hologram strength (which, for weak holograms, is equal to the square root of the saturation diffraction efficiency after sufficient readout) as the approximate $M / \#$. The conventional definition of $M / \#$ is $M / \#=A_{0} \tau_{e}{ }^{\prime} / \tau_{r}$, where $A_{0}, \tau_{e}{ }^{\prime}$, and $\tau_{r}$ are saturation hologram strength, erasure time constant, and recording time constant, respectively. Note that $\tau_{e}{ }^{\prime}$ represents the erasure of a hologram caused by recording other holograms. This erasure in two-center recording is caused by the simultaneous presence of sensitizing and recording beams. The deviation of $M / \#$ from the saturation hologram strength $\left(A_{0}\right)$ is due to the asymmetry between recording and erasure time constants. This asymmetry can occur in crystals (such as $\mathrm{LiNbO}_{3}$ ) in which current is dominated by the bulk photovoltaic effect. The asymmetry is due to the recording dynamics with complex-valued (instead of real-valued) recording time constants. In $\mathrm{LiNbO}_{3}$, it can be seen in highly doped and reduced samples. When this asymmetry occurs, the recording dynamics is not simply a monoexponential function: Its behavior near saturation is oscillatory. The nice monoexponential recording curves obtained in twocenter recording with UV and red light suggests that the dynamics has a real-valued recording time constant. Therefore, little (if any) asymmetry exists between recording and erasure time constants in two-center recording, and $M / \#$ can be approximated by the strength of the saturation hologram. The details of hologram multiplexing in two-center recording can be found in Ref. 38, in which we showed that the difference between (experimentally measured) relevant recording and erasure time constants is $\sim 10 \%$. We also showed that our approximate measure for the $M / \#$ that is computed from single holograms is actually valid for multiplexing of several holograms. ${ }^{38}$

Our focus in this paper has been mainly on $M / \#$ as the holographic performance measure. Another important property to be measured in holographic recording is sensitivity. The sensitivity in a two-center holographic re- 
cording has already been discussed in another publication. $^{39}$ We showed in Ref. 39 that we can improve the sensitivity of two-center recording by a factor of 20 if we use green light instead of red light for recording.

\section{CONCLUSIONS}

Two-center holographic recording is a promising method for persistent holographic recording in $\mathrm{LiNbO}_{3}$ crystals. It is based on using a sensitizing beam and two recording beams to record a hologram in a doubly doped crystal. Both traps are so deep that thermal depopulation of either trap can be neglected. The crystal needs to be annealed properly to ensure that initially all shallower traps as well as a portion of the deeper traps are empty. The success of the method is due to a big asymmetry in the physical mechanisms that are responsible for recording and readout. During recording, an electron undergoes on average many cycles of excitation from traps to the conduction band, moving in the conduction band, and getting trapped. During readout, an electron is limited on average to only a few of these cycles. Therefore, read out causes only a partial erasure of the stored information, and after that the remaining hologram can be read out for a long time without considerable erasure. Although the hologram is initially recorded in both traps, it is finally recorded in the deeper traps after sufficient readout. The presence of UV light during recording is crucial for asymmetry between recording and readout. Without UV light during recording, a strong hologram cannot be recorded.

Initial experimental results suggest that it is possible to obtain $M / \# \simeq 10$ for a 1 -cm-thick $\mathrm{LiNbO}_{3}: \mathrm{Fe}: \mathrm{Mn}$ crystal. $M / \#$ depends mainly on the ratio of the recording and sensitizing intensities. Sensitization and bleaching experiments are helpful for choosing these intensities. For the $\mathrm{LiNbO}_{3}: \mathrm{Fe}: \mathrm{Mn}$ crystal that we used, this ratio was approximately 25-30. It can be optimized by choice of the correct ratio among the excitation and recombination rates of the two traps. This procedure results in the interdependence of the concentrations of the traps, the oxidation-reduction state of the crystal, and the sensitizing and recording intensities. We can obtain the best $M / \#$ by choosing the concentration of the shallower traps to be as large as is practically possible and then optimiz- ing the concentration of the deeper traps and the oxidation-reduction state. As a rule of thumb, for $\mathrm{LiNbO}_{3}: \mathrm{Fe}: \mathrm{Mn}$ the best Mn concentration would be $10 \%$ of that of $\mathrm{Fe}$, and the best annealing state would be found when $\sim 95 \%$ of the Mn traps were filled and all Fe traps were empty. The choice of the sensitizing wavelength is also a crucial step in improving $M / \#$. This wavelength should be long enough to prevent extra absorption owing to band-to-band absorption of $\mathrm{LiNbO}_{3}$ but short enough to provide effective sensitization from the deeper traps. The best sensitizing wavelength for $\mathrm{LiNbO}_{3}: \mathrm{Fe}: \mathrm{Mn}$ crystals is approximately $400-410 \mathrm{~nm}$.

\section{APPENDIX A: DERIVATION OF $\boldsymbol{N}_{\mathrm{Fe}, \text { final }}$}

In this appendix we derive the relationship between the transmitted-to-incident intensities in the sensitization experiment and the final (steady-state) value of the electron concentration in the Fe traps. The complication in the theoretical calculation is due to the variation of $\mathrm{NFe}^{-}$ within the thickness of the crystal (as a result of the large UV absorption). First we rewrite Eq. (21) as

$$
\begin{aligned}
\frac{\partial N_{\mathrm{Fe}}{ }^{-}}{\partial t} & \\
= & -\frac{N_{\mathrm{Fe}}{ }^{-}}{\tau(z)} \\
& +\frac{\gamma_{\mathrm{Fe}} q_{\mathrm{Mn}, \mathrm{UV}} s_{\mathrm{Mn}, \mathrm{UV}} N_{\mathrm{Fe}} N_{A} I_{\mathrm{UV} 0} \exp \left(-\alpha_{\mathrm{UV}} z\right)}{\gamma_{\mathrm{Fe}} N_{\mathrm{Fe}}+\gamma_{\mathrm{Mn}}\left(N_{\mathrm{Mn}}-N_{A}\right)+\left(\gamma_{\mathrm{Mn}}-\gamma_{\mathrm{Fe}}\right) N_{\mathrm{Fe}}{ }^{-}} \\
& +O\left(\left[N_{\mathrm{Fe}}{ }^{-}\right]^{2}\right) \\
= & \frac{N_{\mathrm{Fe}, \mathrm{final}}{ }^{-}-N_{\mathrm{Fe}}{ }^{-}}{\tau(z)},
\end{aligned}
$$

where $\tau(z)$ is the space-dependent sensitization time constant, defined by

$$
\frac{1}{\tau(z)}=\left[\frac{q_{\mathrm{Fe}, \mathrm{UV}} s_{\mathrm{Fe}, \mathrm{UV}} \gamma_{\mathrm{Mn}}\left(N_{\mathrm{Mn}}-N_{A}\right)+\gamma_{\mathrm{Fe}} q_{\mathrm{Mn}, \mathrm{UV}} s_{\mathrm{Mn}, \mathrm{UV}}\left(N_{\mathrm{Fe}}+N_{A}\right)}{\gamma_{\mathrm{Fe}} N_{\mathrm{Fe}}+\gamma_{\mathrm{Mn}}\left(N_{\mathrm{Mn}}-N_{A}\right)+\left(\gamma_{\mathrm{Mn}}-\gamma_{\mathrm{Fe}}\right) N_{\mathrm{Fe}}{ }^{-}}\right] I_{\mathrm{UV} 0} \exp \left(-\alpha_{\mathrm{UV}} z\right) .
$$

Therefore we can express $N_{\mathrm{Fe}}{ }^{-}(z)$ approximately as

$$
N_{\mathrm{Fe}}{ }^{-}(z, t)=N_{\mathrm{Fe}, \text { final }}{ }^{-}\left\{1-\exp \left[-A t \exp \left(-\alpha_{\mathrm{UV}} z\right)\right]\right\},
$$

where

$$
A=\frac{q_{\mathrm{Fe}, \mathrm{UV}} s_{\mathrm{Fe}, \mathrm{UV}}\left[1+\frac{q_{\mathrm{Mn}, \mathrm{UV}} s_{\mathrm{Mn}, \mathrm{UV}}}{q_{\mathrm{Fe}, \mathrm{UV}} s_{\mathrm{Fe}, \mathrm{UV}}} \frac{\gamma_{\mathrm{Fe}} N_{\mathrm{Fe}}\left(1+N_{A} / N_{\mathrm{Fe}}\right)}{\gamma_{\mathrm{Mn}}\left(N_{\mathrm{Mn}}-N_{A}\right)}\right] I_{\mathrm{UV} 0}}{1+\frac{\gamma_{\mathrm{Fe}}\left(N_{\mathrm{Fe}}-N_{\mathrm{Fe}}{ }^{-}\right)}{\gamma_{\mathrm{Mn}}\left(N_{\mathrm{Mn}}-N_{A}\right)}+\frac{N_{\mathrm{Fe}}{ }^{-}}{\left(N_{\mathrm{Mn}}-N_{A}\right)}} .
$$


To calculate the total transmitted red light through the crystal, we we need to include the variation of the absorption coefficient for red light throughout the thickness of the crystal. Considering

$$
I_{t}=I_{i} \exp \left[\int_{0}^{L}-\alpha(z, t) \mathrm{d} z\right]
$$

with

$$
\alpha(z, t)=s_{\mathrm{Fe}, R} h \nu N_{\mathrm{Fe}}{ }^{-}(z, t),
$$

and using Eq. (A3) yield

$$
\begin{aligned}
& -\frac{1}{s_{\mathrm{Fe}, R} h \nu} \ln \left(I_{t} / I_{i}\right) \\
& \quad=N_{\mathrm{Fe}, \mathrm{final}}-\int_{0}^{L}\left\{1-\exp \left[-A t \exp \left(-\alpha_{\mathrm{UV}} z\right)\right]\right\} \mathrm{d} z .
\end{aligned}
$$

We can simplify the calculation of the integral in Eq. (A7) by defining a new variable, $u=A t \exp \left(-\alpha_{\mathrm{UV}} z\right)$. Applying this change of variable results in

$$
\begin{aligned}
\int_{0}^{L}\{1- & \left.\exp \left[-A t \exp \left(-\alpha_{\mathrm{UV}} z\right)\right]\right\} \mathrm{d} z \\
& =L\left[1-\frac{1}{\alpha_{\mathrm{UV}} L} \int_{A t \exp \left(-\alpha_{\mathrm{UV}} L\right)}^{A t} \frac{\exp (-u)}{u} \mathrm{~d} u\right] .
\end{aligned}
$$

To simplify Eq. (A8) further, we need to assume some approximate value for $A$. We first assume a value for $N_{A} / N_{\text {Mn }}$ and substitute it into Eq. (17) to find an approximate value for $\gamma_{\mathrm{Mn}} / \gamma_{\mathrm{Fe}}$. Substituting these approximate values into Eq. (A4), we obtain an approximate value for $A$. We then check the validity of the assumed value for $N_{A} / N_{\mathrm{Mn}}$ by solving the governing equations for $N_{\mathrm{A}}$ [Eqs. (17), (24), and (25)]. After trying different values, we have found that $N_{A} / N_{\mathrm{Mn}} \simeq 0.9$ is a good approximation; it yields $A \simeq 0.01 \mathrm{~s}^{-1}$. For sensitization of $\sim 3 \mathrm{~h}$, we can calculate $A t \simeq 108$. For our $L=0.85$-mm-thick crystal with $\alpha_{\mathrm{UV}}=9 \mathrm{~mm}^{-1}$ at $365 \mathrm{~nm}$, we have $A t \exp \left(-\alpha_{\mathrm{UV}} L\right)$ $\simeq 0.05$. The integral in Eq. (A8) can be calculated by use of the tabulated exponential integral function. The upper bound of this integral can be replaced with $+\infty$. The interesting property of the integral is that it is not a sharp function of the lower bound (and, therefore, of $A$ ) in the range of values that are relevant to our experiment. For example, for lower bounds $\left[A t \exp \left(-\alpha_{\mathrm{UV}} L\right)\right]$ of 0.04 , 0.05 , and 0.07 , the integral is equal to $0.65 \mathrm{~L}, 0.68 \mathrm{~L}$, and $0.72 L$, respectively. This shows that the method that we have followed here to obtain an approximate value for $A$ does not significantly affect the final result for $N_{\mathrm{Fe} \text {,final }}{ }^{-}$. Using $0.7 L$ as the value of the integral in Eq. (A7), and using $I_{t} / I_{i}=0.9$ from Fig. $7(\mathrm{~b})$, we obtain $N_{\mathrm{Fe} \text {,final }}$ $\simeq 1.16 \times 10^{24} \mathrm{~m}^{-3}$.

\section{ACKNOWLEDGMENTS}

This research was supported by a Jet Propulsion Laboratory work order funded by the Defense Advanced Research Projects Agency-ITO and by Air Force/Rome Lab award F0060297C0049. A National Science Foundation/ Deutscher Akademischer Austauschdienst grant for joint research is gratefully appreciated.

*Present address, School of Electrical and Computer Engineering, Georgia Institute of Technology, Atlanta, Georgia 30332; e-mail address, adibi@ee.gatech.edu.

${ }^{\dagger}$ Present address, Physical Department, University of Bonn, Wegelerstrasse 8, D-53115 Bonn, Germany.

\section{REFERENCES}

1. F. H. Mok, "Angle-multiplexed storage of 5000 holograms in lithium niobate," Opt. Lett. 18, 915-917 (1993).

2. I. McMichael, W. Christian, D. Pletcher, T. Y. Chang, and J. H. Hong, "Compact holographic storage demonstrator with rapid access," Appl. Opt. 35, 2375-2379 (1996).

3. J. Ashley, M.-P. Bernal, M. Blaum, G. W. Burr, H. Coufal, R. K. Grygier, H. Günter, J. A. Hoffnagle, C. M. Jefferson, R. M. MacFarlane, B. Marcus, R. M. Shelby, G. T. Sincerbox, and G. Wittmann, "Holographic storage promises high data density," Laser Focus World, April 1996, pp. 81-93.

4. J. J. Amodei and D. L. Staebler, "Holographic pattern fixing in electro-optic crystals," Appl. Phys. Lett. 18, 540-542 (1971).

5. F. Micheron and G. Bismuth, "Electrical control of fixation and erasure of holographic patterns in ferroelectric materials," Appl. Phys. Lett. 20, 79-81 (1972).

6. D. von der Linde, A. M. Glass, and K. F. Rodgers, "Multiphoton photorefractive processes for optical storage in $\mathrm{LiNbO}_{3}$," Appl. Phys. Lett. 25, 155-157 (1974).

7. R. A. Rupp, H. C. Külich, U. Schürk, and E. Krätzig, "Diffraction by difference holograms in electrooptic crystals," Ferroelectrics 8, 25-30 (1987).

8. H. C. Külich, "A new approach to read volume holograms at different wavelengths," Opt. Commun. 64, 407-411 (1987).

9. H. Vormann and E. Krätzig, "Two step excitation in $\mathrm{LiTaO}_{3}: \mathrm{Fe}$ for optical data storage," Solid State Commun. 49, 843-847 (1984).

10. K. Buse, F. Jermann, and E. Krätzig, "Two-step photorefractive hologram recording in $\mathrm{LiNbO}_{3}: \mathrm{Fe}$," Ferroelectrics 141, 197-205 (1993).

11. K. Buse, F. Jermann, and E. Krätzig, "Infrared holographic recording in $\mathrm{LiNbO}_{3}: \mathrm{Cu}$," Appl. Phys. A 58, 191-195 (1994).

12. K. Buse, F. Jermann, and E. Krätzig, "Infrared holographic recording in $\mathrm{LiNbO}_{3}: \mathrm{Fe}$ and $\mathrm{LiNbO}_{3}: \mathrm{Cu}$," Opt. Mater. 4, 237-240 (1995).

13. N. Iyi, K. Kitamura, F. Izumi, J. K. Yamamoto, T. Hayashi, H. Asano, and S. Kimura, "Comparative study of defect structures in lithium niobate with different compositions," J. Solid State Chem. 101, 340-352 (1992).

14. N. Zotov, H. Boysen, J. Schneider, and F. Frey, "Application of combined neutron and x-ray powder diffraction refinements to the structure of congruent lithium niobate," Mater. Sci. Forum 166-169, 631-636 (1994).

15. Y. S. Bai, R. R. Neurgaonkar, and R. Kachru, "Resonant two-photon photorefractive grating in praeseodymiumdoped strontium barium niobate with cw lasers," Opt. Lett. 21, 567-569 (1996).

16. Y. S. Bai and R. Kachru, "Nonvolatile holographic storage with two-step recording in lithium niobate using cw lasers," Phys. Rev. Lett. 78, 2944-2947 (1997).

17. H. Guenther, G. Wittmann, R. M. Macfarlane, and R. R. Neurgaonkar, "Intensity dependence and white-light gating of two-color photorefractive gratings in $\mathrm{LiNbO}_{3}$," Opt. Lett. 22, 1305-1307 (1997).

18. H. Guenther, R. Macfarlane, Y. Furukawa, K. Kitamura, and R. Neurgaonkar, "Two-color holography in reduced 
near-stoichiometric lithium niobate," Appl. Opt. 37, 76117623 (1998)

19. L. Hesselink, S. S. Orlov, A. Liu, A. Akella, D. Lande, and R. R. Neugaonkar, "Photorefractive materials for nonvolatile volume holographic data storage," Science 282, 10891094 (1998).

20. K. Buse, A. Adibi, and D. Psaltis, "Non-volatile holographic storage in doubly doped lithium niobate crystals," Nature 393, 665-668 (1998).

21. A. Adibi, K. Buse, and D. Psaltis, "Hologram multiplexing using two-step recording," in Advanced Optical Memorials and Interfaces to Computer Storage, Z. U. Hasan and P. A. Mitkas, ed., Proc. SPIE 3468, 20-29 (1998).

22. D. L. Staebler and W. Phillips, "Hologram storage in photochromic $\mathrm{LiNbO}_{3}$," Appl. Phys. Lett. 24, 268-270 (1974).

23. O. Thiemann and O. F. Schirmer, "Energy levels of several 3d impurities and EPR of $\mathrm{Ti}^{3+}$ in $\mathrm{LiNbO}_{3}$," in Electro-Optic and Magneto-Optic Materials, J.-P. Huignard, ed., Proc. SPIE 1018, 18-22 (1988).

24. W. Phillips, J. J. Amodei, and D. L. Staebler, "Optical and holographic storage properties of transition metal doped lithium niobate," RCA Rev. 33, 94-109 (1972).

25. F. Jermann and J. Otten, "Light-induced charge transport in $\mathrm{LiNbO}_{3}: \mathrm{Fe}$ at high light intensities," J. Opt. Soc. Am. B 10, 2085-2092 (1993).

26. R. T. Smith and F. S. Welsh, "Temperature dependence of the elastic, piezoelectric, and dielectric constants of lithium tantalate and lithium niobate," J. Appl. Phys. 42, 2219 2230 (1971).

27. A. Mansingh and A. Dhar, "The ac conductivity and dielectric constant of lithium niobate single crystals," J. Phys. D 18, 2059-2071 (1985).

28. K. Onuki, N. Uchida, and T. Saku, "Interferometric method for measuring electro-optic coefficients in crystals," J. Opt. Soc. Am. 62, 1030-1032 (1972).
29. G. J. Edwards and M. Lawrence, "A temperaturedependent dispersion equation for congruently grown lithium niobate," Opt. Quantum Electron. 16, 373-375 (1984).

30. Y. Ohmori, M. Yamaguchi, K. Yoshino, and Y. Inuishi, "Electron hall mobility in reduced $\mathrm{LiNbO}_{3}$," Jpn. J. Appl. Phys. 15, 2263-2264 (1976).

31. H. Kurz, E. Krätzig, W. Keune, H. Engelmann, U. Gonser, B. Dischler, and A. Räuber, "Photorefractive centers in $\mathrm{LiNbO}_{3}$, studied by optical, Mössbauer, and EPR methods," Appl. Phys. (N.Y.) 12, 355-368 (1977).

32. E. Krätzig and H. Kurz, "Photo-induced currents and voltages in $\mathrm{LiNbO}_{3}$," Ferroelectrics 13, 295-296 (1976).

33. E. Krätzig and H. Kurz, "Photorefractive and photovoltaic effects in doped $\mathrm{LiNbO}_{3}$," Opt. Acta 24, 475-482 (1977).

34. H. Kogelnik, "Coupled wave theory for thick hologram gratings,” Bell Syst. Tech. J. 48, 2909-2947 (1969).

35. F. H. Mok, G. W. Burr, and D. Psaltis, "System metric for holographic memory systems," Opt. Lett. 21, 896-898 (1996).

36. R. Orlowski and E. Krätzig, "Holographic method for the determination of photoinduced electron and hole transport in electro-optic crystals," Solid State Commun. 27, 13511354 (1978).

37. A. Adibi, K. Buse, and D. Psaltis, "Effect of annealing in two-center holographic recording," Appl. Phys. Lett. 74 3767-3769 (1999).

38. A. Adibi, K. Buse, and D. Psaltis, "Multiplexing holograms in $\mathrm{LiNbO}_{3}: \mathrm{Fe}: \mathrm{Mn}$ crystals," Opt. Lett. 24, 652-654 (1999).

39. A. Adibi, K. Buse, and D. Psaltis, "Sensitivity improvement in two-center holographic recording," Opt. Lett. 25, 539$541(2000)$. 